\title{
A better characterization of the chemical composition of exoplanets atmospheres with ARIEL
}

\author{
Olivia Venot • Benjamin Drummond . \\ Yamila Miguel • Ingo P. Waldmann • Enzo \\ Pascale . Tiziano Zingales
}

Received: date / Accepted: date

\begin{abstract}
Since the discovery of the first extrasolar planet more than twenty years ago, nearly four thousand planets orbiting stars other than the Sun ${ }^{1}$ have been discovered. Current observational instruments (on board the Hubble Space Telescope, Spitzer, and on ground-based facilities) have allowed the scientific community to obtain important information on the physical and chemical properties of these planets. However, for a more in-depth characterisation of these worlds, more powerful telescopes are needed. Thanks to the high sensitivity of their instruments, the next generation of space observatories (e.g. JWST, ARIEL) will provide observations of unprecedented quality, allowing us to extract far more information than what was previously possible. Such high quality observations will provide constraints on theoretical models of exoplanet atmospheres and lead to a greater

O. Venot

Laboratoire Interuniversitaire des Systèmes Atmosphériques, UMR CNRS 7583, Université Paris Est Créteil (UPEC) et Université Paris Diderot (UPD), Institut Pierre Simon Laplace (IPSL), Créteil, France

E-mail: olivia.venot@lisa.u-pec.fr

B. Drummond

Astrophysics Group, University of Exeter, EX4 4QL, Exeter, UK

Y. Miguel

Laboratoire Lagrange, UMR 7293, Université de Nice-Sophia Antipolis, CNRS, Observatoire de la Côte d'Azur, Blvd de l'Observatoire, CS 34229, 06304 Nice cedex 4, France, and

Leiden Observatory, University of Leiden, Niels Bohrweg 2, 2333CA Leiden, The Netherlands

I. P. Waldmann

University College London, Department of Physics and Astronomy, Gower Street, London WC1E 6BT, UK

E. Pascale

Dipartimiento di Fisica, La Sapienza Università di Roma, Piazzale Aldo Moro 2, 00185 Roma, Italy, and

Cardiff University, School of Physics and Astronomy, 5 The Parade, Cardiff, CF24 3AA, UK

T. Zingales

University College London, Department of Physics and Astronomy, Gower Street, London WC1E 6BT, UK, and

INAF-Osservatorio Astronomico di Palermo, Piazza del Parlamento 1, I-90134 Palermo, Italy

1 http://exoplanet.eu/
\end{abstract}


understanding of their physics and chemistry. Important modelling efforts have been carried out during the past few years, showing that numerous parameters and processes (such as the elemental abundances, temperature, mixing, etc.) are likely to affect the atmospheric composition of exoplanets and subsequently the observable spectra. In this manuscript, we review the different parameters that can influence the molecular composition of exoplanet atmospheres. We show that the high-precision of ARIEL observations will improve our view and characterisation of exoplanet atmospheres. We also consider future developments that are necessary to improve atmospheric models, driven by the need to interpret the available observations.

Keywords Atmospheres · Exoplanets - Composition · Modelling · Laboratory measurements · Observations

\section{Introduction}

The number of known exoplanets has exploded during the past few years. It is now clear that there exists a huge diversity of worlds in terms of radius, mass, temperature, orbital eccentricity, etc. This wealth of new information has implications for planetary formation scenarios, originally formulated in the time when only the Solar system planets were known. The chemical composition of the atmosphere is the key to understanding planetary formation. In particular, the elemental composition is expected to depend on the environment in which the planet formed, and through which it evolved. Thanks to spectroscopic observations performed during planetary transits, the atmosphere of planets can be probed, giving access to their chemical composition.

To a fundamental level, the chemical composition of the atmosphere is determined by 1) the elemental abundances the planet formed with and 2) the temperature of the atmosphere, which is dependent on irradiation and internal heating. In addition, physical processes such as mixing, photolysis, and atmospheric escape, occur in different pressure regions of exoplanet atmospheres (see Fig. 1 of Madhusudhan et al 2016), and can influence the atmospheric composition, with subsequent effects on the observable spectra. Infrared (IR) observations probe the middle atmosphere $\left(\sim 0.1-10^{-5}\right.$ bar $)$, giving information on the abundances of neutral species, whereas ultraviolet (UV) observations are used to study the upper atmosphere, where atomics species and ions are highly present. Models that couple chemical kinetics to the out of equilibrium processes mentioned above are used to predict the chemical abundances and to understand the spectroscopic observations.

Several models have been developed up to now (e.g. Liang et al 2003, 2004, Koskinen et al |2007; Zahnle et al |2009a|b; Line et al |2010,| Moses et al||2011 | $\mathrm{Hu}$ et al 2012 | Kopparapu et al 2012 |Venot et al 2012; Agúndez et al 2012; Lavvas et al 2014 Miguel and Kaltenegger 2014 Drummond et al 2016; Rimmer and Helling 2016 Tsai et al 2017) which differ in the complexity, size (number of species and reactions) and type (neutral/ionic) of chemistry, the temperature range over which the chemical scheme is valid, and the atmospheric layers that can be studied with (e.g. middle atmosphere, upper atmosphere). In this manuscript, we focus on the chemistry of neutral species. Depending on their abundance, these latter will have spectral signatures in the future observations of ARIEL (Atmospheric Remotesensing Exoplanet Large-survey). 
Among the different categories of exoplanets, the warm gaseous giant planets are the most interesting. Firstly, they provide the highest quality observations that are necessary for model-observation comparisons. Secondly, the abundances of the major molecules $\left(\mathrm{H}_{2} \mathrm{O}, \mathrm{CO}, \mathrm{NH}_{3}\right.$, etc. $)$ constrained by observations might be a direct reflection of the elemental abundances of the planetary atmosphere, unlike giant planets of the Solar System. Indeed, the abundance of oxygen in the atmosphere of Jupiter or Uranus is highly uncertain because the condensation of water removes oxygen species from the observable part of the atmosphere (Cavalié et al 2017). However, in the much hotter atmospheres of short-period exoplanets, condensation is limited to high temperature condensate species, such as $\mathrm{TiO}$ or VO, which are not the main carrier of elemental oxygen. Finally, the atmospheres of gas giants may be similar to the elemental composition with which they formed; unlike smaller planets which may be affected by delivery from meteorites/comets or out-gassing from the rocky interior. We precise that determining the enrichment of the planetary atmosphere relative to the elemental composition of the star requires in addition good observations of the host star.

The goal of this manuscript is to consider some important model parameters that effect the atmospheric composition and, most importantly, to test whether these effects are significant enough to be detected with ARIEL. We consider several previously published studies and calculate new synthetic transmission and emission spectra, taking into account the technical characteristics of the instruments on board ARIEL.

In Section 2, we present the tools that have been used in this paper: a series of one-dimensional kinetic models, a forward radiative-transfer code and the ARIEL noise model. In Section 3 we describe the difference between equilibrium and disequilibrium chemistry regimes. In Sections 4, 5 and 6 we consider how various planetary, orbital and stellar parameters effect the synthetic spectra, specifically in the context of ARIEL. Finally, we look forward to potential advances in understanding that will be achieved with the ESA ARIEL space mission.

\section{Models used in this work}

\subsection{One-dimensional chemical kinetics models}

The results presented in this study are obtained using a number of different chemical models. These one-dimensional, time-dependent models include chemical kinetics as well as processes that drive the chemistry away from local chemical equilibrium (vertical mixing and photochemistry). These models are fully described in: Venot et al (2012), Miguel and Kaltenegger (2014), Agúndez et al (2014a) and Drummond et al (2016).

Generally, these models represent the atmosphere as a vertical column with a given thermal profile. This profile is typically divided into several layers with a thickness equal to a constant fraction of the pressure scale height; except for Drummond et al (2016) where the layers are determined directly by hydrostatic balance. To determine the chemical composition of the column, the continuity equation (Eq. 1) is solved for the steady-state of each species in each model level,

$$
\frac{\partial n_{i}}{\partial t}=P_{i}-n_{i} L_{i}-\operatorname{div}\left(\Phi_{i} \overrightarrow{e_{z}}\right)
$$


where $n_{i}$ the number density $\left(\mathrm{cm}^{-3}\right), P_{i}$ the production rate $\left(\mathrm{cm}^{-3} \mathrm{~s}^{-1}\right), L_{i}$ the loss rate $\left(\mathrm{s}^{-1}\right)$, and $\Phi_{i}$ the vertical flux $\left(\mathrm{cm}^{-2} \mathrm{~s}^{-1}\right)$, respectively, of the species $i$. The vertical flux is parameterised by the vertical diffusion equation,

$$
\Phi_{i}=-n_{i} D_{i}\left[\frac{1}{n_{i}} \frac{\partial n_{i}}{\partial z}+\frac{1}{H_{i}}+\frac{1}{T} \frac{d T}{d z}\right]-n_{i} K\left[\frac{1}{y_{i}} \frac{\partial y_{i}}{\partial z}\right],
$$

where $K$ is the eddy diffusion coefficient $\left(\mathrm{cm}^{2} \mathrm{~s}^{-1}\right), D_{i}$ is the molecular diffusion coefficient $\left(\mathrm{cm}^{2} \mathrm{~s}^{-1}\right), H_{i}$ the scale height, and $y_{i}$ the mixing ratio of the species $i$. At both upper and lower boundaries, a zero flux for each species is usually imposed.

One of the main ingredients of a kinetics model is the chemical network which, in essence, is a list of chemical reactions and associated rate constants. Venot et al (2012) implemented a chemical network entirely new in planetology, specifically adapted to the extreme conditions of warm exoplanet atmospheres. This scheme has been developed in close collaboration with experts in the combustion industry. Its strength comes from its global experimental validation that has been performed across a large range of pressures (0.01-100 bar) and temperatures (300-2500 K). The network describes the kinetics of species made of $\mathrm{H}, \mathrm{C}, \mathrm{O}$, and $\mathrm{N}$, including hydrocarbons with up to two carbon atoms. The 105 compounds of this scheme are linked by $\sim 2000$ reactions. All the results presented in this paper have been found using this chemical scheme (i.e. Venot et al 2014, 2015, 2016, 2018, Agúndez et al 2012, 2014a b; Drummond et al 2016) except results of Miguel and Kaltenegger (2014) and Miguel et al (2015) that have been found using a smaller chemical scheme of 19 species and 179 reactions.

In order to study atmospheres rich in carbon, where hydrocarbons can be abundant and thus have a non-negligible influence on the chemical composition, Venot et al (2015) developed an extended version of the chemical network, able to describe kinetics of species containing up to six carbon atoms. This large scheme contains 240 species and $\sim 4000$ reactions. Both of these schemes are available on the KIDA Database ${ }^{2}$ (Wakelam et al 2012).

\subsection{Radiative transfer model}

In this study we calculate a series a transmission and emission spectra using the radiative transfer forward model in the TauREx retrieval framework (Waldmann et al 2015b a), using thermal and composition profiles from previously published works. The infrared absorption cross-sections of the absorbing species were computed using the linelists from ExoMol (Tennyson and Yurchenko 2012), where available, and otherwise taken from HITEMP (Rothman et al 2010) and HITRAN (Rothman et al 2009, 2013). Cross sections and subsequent forward models were computed at a resolution of $\mathrm{R}=7,000$ (constant in wavelength). We assume 300 plane-parallel atmospheric layers and cloud-free atmospheres. To simulate the observations that ARIEL will provide, we bin the high-resolution forward model to the wavelength and resolution grid of ARIEL for the specified signal-to-noise of the observation (see Section 2.3).

\footnotetext{
2 http://kida.obs.u-bordeaux1.fr/
} 


\subsection{ARIEL noise model}

The signal-to-noise ratio (SNR) has been evaluated using a radiometric model which implements a detailed description of the ARIEL instrument design described in details in the ARIEL Assessment Study Report (Yellow Book) ${ }^{3}$

The noise model implemented provides an accurate estimate of the experimental uncertainties of the planet spectra ARIEL will measure. All major sources of photometric uncertainty are accounted for. Among these are: photodetector non uniform response; intra-pixel variations; pointing stability; photon shot noise; detector read noise. Models implemented are cross validated using the end-to-end time-domain transit-spectroscopy simulator ExoSim (Sarkar et al 2016), and using the radiometric model developed by ESA (Puig et al 2015$)$. Details of the cross-validation of these simulation tools are found in the ARIEL Yellow Book accompanying documentation.

In Table 1 we show the spectral coverage of ARIEL and the nominal resolution of each band. In Table 2 we present the SNR per transit of the three planets studied in this paper.

Table 1 ARIEL spectral coverage and nominal resolution. The three first channels are photometric bands. The three last ones are covered by ARIEL spectrograph.

\begin{tabular}{c|cc}
\hline \hline Spectral range & Wavelength range $(\mu \mathrm{m})$ & Resolution \\
\hline 1 & $0.5-0.55$ & Integrated band \\
\hline 2 & $0.8-1.0$ & Integrated band \\
\hline 3 & $1.05-1.2$ & Integrated band \\
\hline \hline 4 & $1.25-1.95$ & 10 \\
\hline 5 & $1.95-3.90$ & 100 \\
\hline 6 & $3.90-7.80$ & 30 \\
\hline
\end{tabular}

Table 2 SNR per transit for the planets of this study.

\begin{tabular}{c|ccc}
\hline \hline Spectral range & HD 189733b & GJ 436b & GJ 3470b \\
\hline 1 & 21.79 & 4.19 & 2.35 \\
\hline 2 & 48.96 & 19.77 & 10.07 \\
\hline 3 & 39.19 & 18.13 & 9.01 \\
\hline \hline 4 & 34.99 & 21.19 & 10.10 \\
\hline 5 & 7.34 & 4.89 & 2.33 \\
\hline 6 & 6.87 & 5.02 & 2.30 \\
\hline
\end{tabular}

\section{The transition between chemical equilibrium and disequilibrium}

Intense irradiation of short-period exoplanets leads to very high atmospheric temperatures of typically $T>1000 \mathrm{~K}$. This might initially suggest that the chemical

\footnotetext{
3 http://sci.esa.int/cosmic-vision/59109-ariel-assessment-study-report-yellow-book/
} 
composition of these atmospheres could be described by thermochemical equilibrium, as such temperatures lead to fast chemical kinetics. The first models used to study exoplanet atmospheres assumed chemical equilibrium (e.g. Burrows and Sharp 1999, Seager and Sasselov 2000; Sharp and Burrows 2007; Barman||2007; Burrows et al 2007, 2008). However, it was quickly realised that such assumptions were not favoured by observations.

Physical processes, such as mixing and photodissociations, can influence the chemical composition (e.g. Zahnle et al 2009b Line et al 2010, Moses et al| 2011, Venot et al 2012, Miguel and Kaltenegger 2014 Drummond et al 2016$)$. In the presence of vertical mixing, a competition exists between the chemical and mixing (or dynamical) processes. If the timescale of the chemistry is faster than that of the mixing, then the atmosphere will achieve chemical equilibrium. On the other hand, if the mixing timescale is faster than the chemical timescale then the atmosphere will be driven away from chemical equilibrium. The pressure level at which these two timescales are equal is usually called the quenching point (i.e. Prinn and Barshay 1977), and the equilibrium abundances at the quench determine the "quenched abundances" for all lower pressures.

It is obvious that depending on the balance of the dynamical and chemical timescales, the composition of the atmosphere can be altered. If processes like mixing and photochemistry lead to significant changes in the abundances of absorbing chemical species, they may lead to signatures in the observable spectra. In this case, photochemical models are required to successfully interpret the spectra and to constrain the chemical composition.

We determined in which cases the atmosphere is likely to be in a state of chemical equilibrium, for a series of different thermal profiles published in Venot et al (2015). These thermal profiles have been computed with the analytical model of Parmentier and Guillot (2014). The three thermal profiles have each an isothermal part of 500,1000 , or $1500 \mathrm{~K}$ in the upper atmosphere, which correspond to irradiation temperatures of 784,1522 , or $2303 \mathrm{~K}$, respectively (see Venot et al 2015 and Parmentier and Guillot 2014 for more details).

For each PT profile, we compared the steady-state abundances of the main species (i.e. $\mathrm{H}_{2}, \mathrm{H}, \mathrm{H}_{2} \mathrm{O}, \mathrm{CH}_{4}, \mathrm{CO}, \mathrm{CO}_{2}, \mathrm{~N}_{2}, \mathrm{NH}_{3}, \mathrm{HCN}, \mathrm{CH}_{3}$, and $\mathrm{OH}$ ) with their thermochemical equilibrium abundances and determined their quenching levels. The deeper quench level defines the equilibrium/disequilibrium limit. Above this level, at least one species is not at thermochemical equilibrium. We reported the pressure and temperature of this quench point for each PT profile on Fig. 1. We also studied how the equilibrium/disequilibrium limit varies with vertical mixing strength, parameterised by the eddy diffusion coefficient $K_{z z}$, which typically varies between $10^{3}<K_{z z}<10^{12} \mathrm{~cm}^{2} \mathrm{~s}^{-1}$.

Figure 1 shows the transition between chemical equilibrium and disequilibrium as a function of pressure and temperature, for different values of $K_{z z}$. Given that the pressure range probed by observations is approximately from 1 to $10^{3}$ mbar, exoplanets with a temperature higher than $1500 \mathrm{~K}$ (in this pressure region) are more likely to be in chemical equilibrium, which, as we said previously, correspond to planets with an irradiation temperature of about $2300 \mathrm{~K}$. For cooler planets disequilibrium is important and the use of a chemical kinetics model (accounting for vertical mixing) is necessary to determine the chemical composition of the atmosphere. 


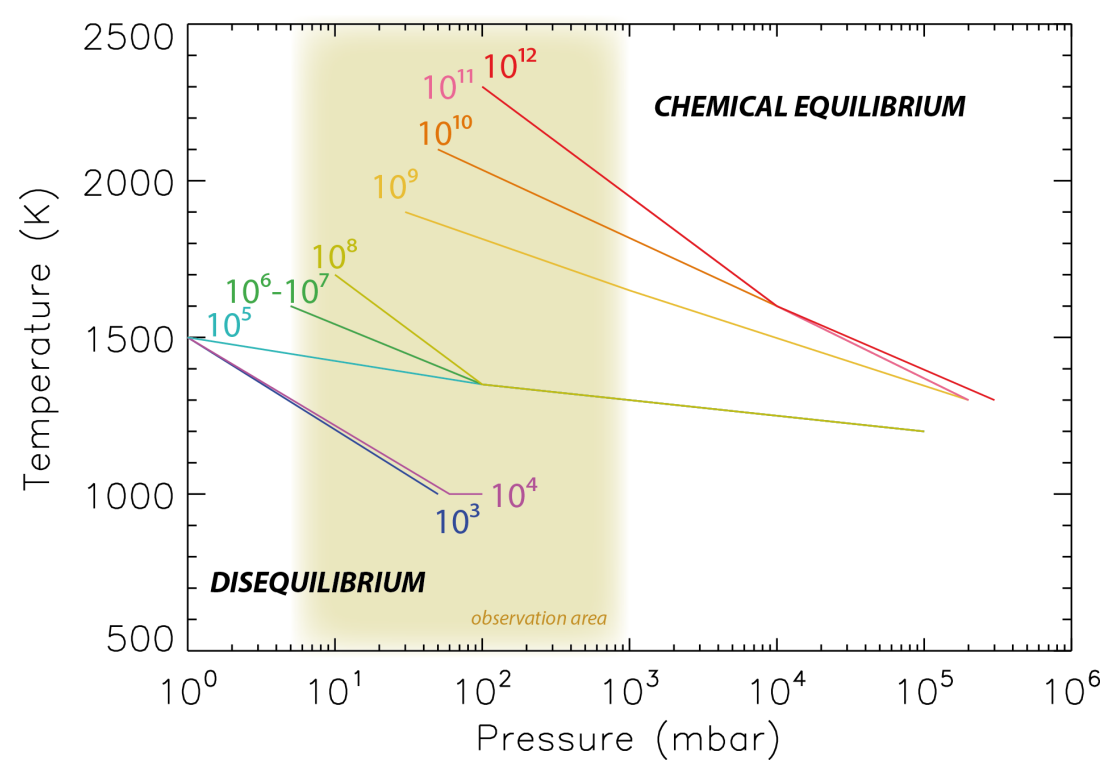

Fig. 1 Pressure-temperature chemical equilibrium/disequilibrium lines calculated for different vertical mixing strengths. The value of the eddy diffusion coefficient (in $\mathrm{cm}^{2} \mathrm{~s}^{-1}$ ) are labeled on the figure and are represented by different colors. For instance, an atmospheric layer at $1500 \mathrm{~K}$ and $10^{2} \mathrm{mbar}$ is at chemical equilibrium if the vertical mixing is lower than $10^{9} \mathrm{~cm}^{2} \mathrm{~s}^{-1}$.

This evaluation is approximate because the equilibrium/disequilibrium transition depends on the value of $K_{z z}$, which is very uncertain. Nevertheless, eddy diffusion coefficients of about $10^{8} \mathrm{~cm}^{2} \mathrm{~s}^{-1}$ are commonly accepted in the community of exoplanets, based on calculations made with 3D models and parametrised to 1D calculations (Parmentier et al 2013). The transition location depends also on the shape of the thermal profile. Two different carbon-to-oxygen ratios $(\mathrm{C} / \mathrm{O})$ have been tested $(\mathrm{C} / \mathrm{O}$ solar and $\mathrm{C} / \mathrm{O}=1.1)$, but this parameter has a negligible effect on the location of the equilibrium/disequilibrium transition.

\section{Influence of planetary and orbital parameters}

4.1 Vertical mixing, metallicity, temperature

For warm planets whose atmospheres are unlikely to be described by chemical equilibrium, it is interesting to quantify the effect of the different parameters that are likely to influence the chemical composition. Venot et al $(2014)$ studied the atmospheric composition of the warm Neptune GJ 3470b. They explored the parameter space for metallicity $(\zeta)$, temperature $(T)$, eddy diffusion coefficient $\left(K_{z z}\right)$, and stellar UV flux $\left(F_{\lambda}\right)$. They varied these parameters with respect to the values of their standard mode 4

\footnotetext{
4 Nominal $\zeta$ is $10 \times$ solar metallicity and nominal $F_{\lambda}, T$, and $K_{z z}$ are represented on their Figs. 2 and 3 respectively.
} 
Venot et al (2014) found that the value of the eddy diffusion coefficient and the intensity of stellar UV irradiation have a lower impact on the chemical composition, compared to the much larger effect of metallicity and temperature, which lead to changes of several orders of magnitude in the abundance of some species.
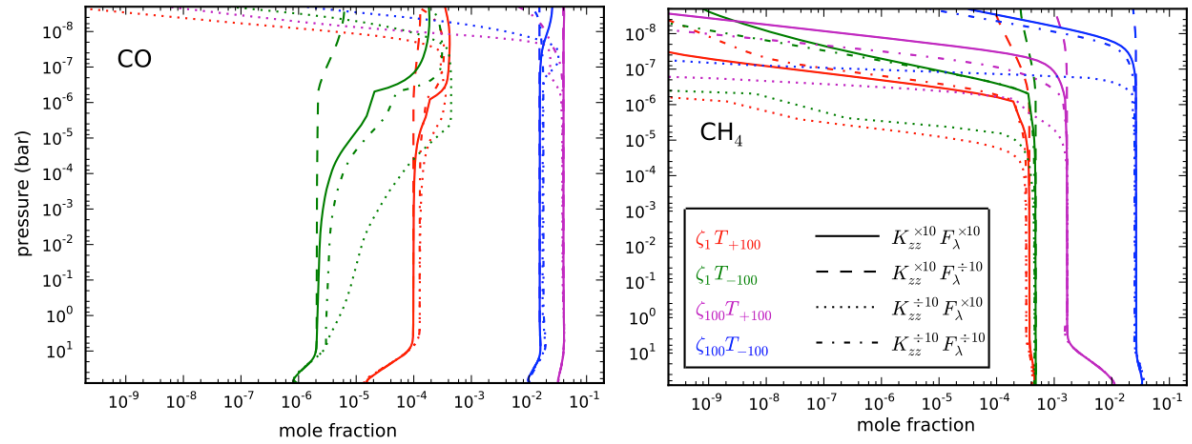

Fig. 2 Vertical abundances profiles of $\mathrm{CO}$ (left) and $\mathrm{CH}_{4}$ (right) from 16 models of GJ 3470b with various values of the metallicity $(\zeta)$, temperature $(T)$, eddy diffusion coefficient $\left(K_{z z}\right)$, and stellar UV flux $\left(F_{\lambda}\right)$. Each color corresponds to a set of metallicity and temperature, and each line style to a set of eddy diffusion coefficient and stellar irradiation. Adapted from Venot et al (2014), reproduced with permission (C)ESO.

Fig. 2 shows the vertical abundance profiles of $\mathrm{CO}$ and $\mathrm{CH}_{4}$, the main reservoirs of carbon, for a range of values of the four parameters described above. In this case, it is apparent that metallicity and temperature are much more important in determining the mole fractions of these two species, compared with the eddy diffusion coefficient and the UV flux.

Fig. 3 shows the transmission spectra for four parameter combinations of metallicity and temperature, each with $K_{z z}^{\times 10} F_{\lambda}^{\times 10}$, including simulated ARIEL observations. All of the spectra have roughly the shame shape, which is dominated by absorption features of $\mathrm{H}_{2} \mathrm{O}$ and $\mathrm{CH}_{4}$. The effect of changing the metallicity and temperature is to vertically shift spectra, since both parameters lead to changes in the atmospheric scale height (Venot et al 2014). The spectra corresponding to the different $\zeta_{x} T_{y}$ cases can be easily differentiated with a signal-to-noise ratio (SNR) of 2 , corresponding to one single transit.

Fig. 4 shows the effect of different eddy diffusion coefficients and UV fluxes on the transmission spectra. For a given $\zeta_{x} T_{y}$, the effect of different vertical mixing and irradiation intensities is quite moderate. For all $\zeta_{x} T_{y}$ cases, we observe variations between 5 and $8 \mu \mathrm{m}$, which are due to changes in $\mathrm{H}_{2} \mathrm{O}$ and $\mathrm{CH}_{4}$ abundances. We notice variations in the $\mathrm{CO}_{2}$ signature around $4.3 \mu \mathrm{m}$ for all the spectra of the high metallicity $\left(\zeta_{100}\right)$ models. For the models with a lower metallicity (i.e. solar, $\left.\zeta_{1}\right)$, this feature appears clearly for the models with a high irradiation and a low vertical mixing $\left(K_{z z}^{\div 10} F_{\lambda}^{\times 10}\right)$ but is absent (or very small) for the three other ones. For these former models $\left(\zeta_{1}\right.$ and $\left.K_{z z}^{\dot{1} 10} F_{\lambda}^{\times 10}\right)$, we also observe a decrease of the transit depth in the $\mathrm{CH}_{4}$ bands between 3 and $4 \mu \mathrm{m}$ compared to the three other models with the same metallicity and temperature. Finally, an important variation that can be observed concerns the spectra of the $\zeta_{100} T_{-100}$ models which 
are shifted vertically depending on the vertical mixing. The $K_{z z}^{\times 10}$ spectra present, on the entire wavelength range, a larger transit depth than the $K_{z z}^{\dot{*} 10}$ ones. This is due to the higher atmospheric scale height of these models. For each $\zeta_{x} T_{y}$ cases, a high SNR $(\geq 20)$ is necessary to differentiate the four spectra corresponding to the different $K_{z z} F_{\lambda}$ cases. Given the SNR per transit for GJ3470b (see Table 2), a SNR of 20 is achievable by combining multiple transits.

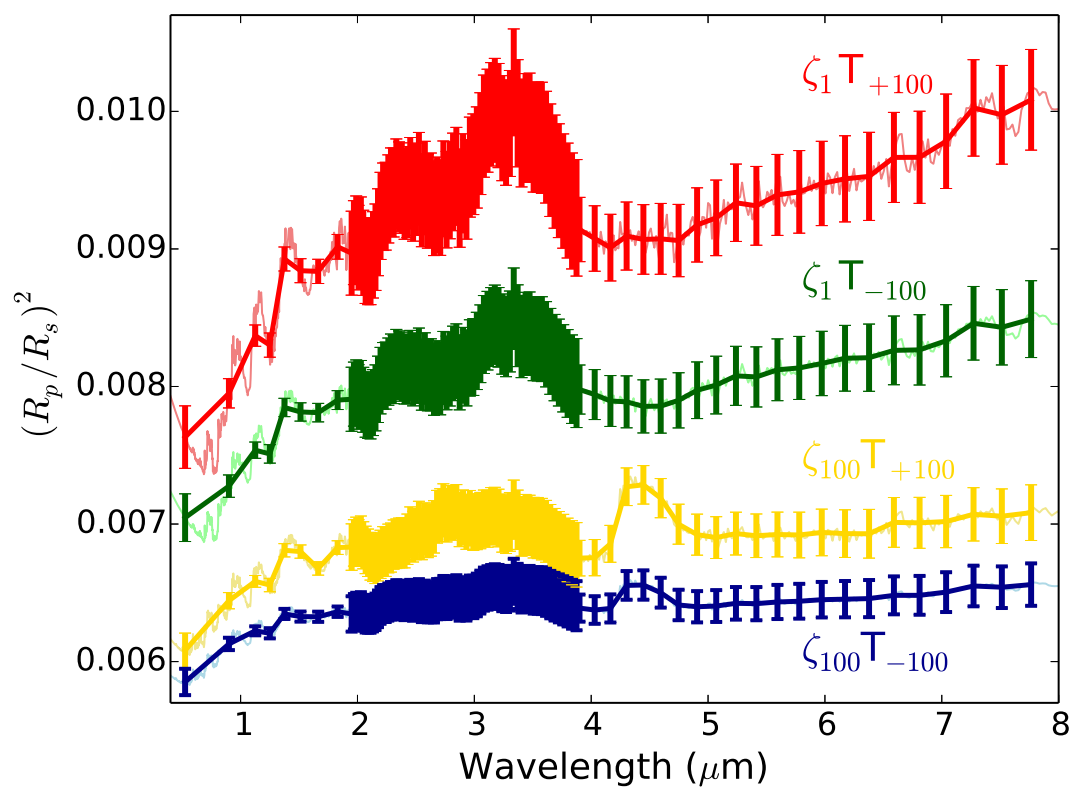

Fig. 3 Synthetic transmission spectra from 4 selected models of GJ 3470b with a high vertical mixing $\left(K_{z z}^{\times 10}\right)$ and a high irradiation $\left(F_{\lambda}^{\times 10}\right)$ but with different metallicities $(\zeta)$ and temperatures $(T)$. The meaning of the different colors is explained on each plot. Bold curves are spectra binned to ARIEL resolution, as explained in Table 1] The error bars correspond to a SNR of 2. Fainter curves are higher-resolution spectra ( $\mathrm{R}=300$, constant in wavelength).

\subsection{Eccentricity}

The effect of orbital eccentricity on the thermal profile and composition was investigated by Agúndez et al (2014b) for the eccentric warm Neptune GJ 436b.

The dissipation of tidal forces in the planet releases an internal heat flow $\phi_{\text {int }}$. To quantify the effect of this heat flow on the thermal structure of the atmosphere, it can be incorporated into a radiative-convective model as an internal temperature $T_{\text {int }}=\left(\phi_{\text {int }} / \sigma\right)^{1 / 4}$, where $\sigma$ is the Stefan-Boltzmann constant. However, it is very challenging to evaluate precisely the dissipation rate of tidal forces in a planet, as it depends on the internal composition and structure, unknown in the case of GJ 436b. To study the tidal forces in GJ 436b, Agúndez et al (2014a) used the Constant Time Lag (CTL) model with the factor $Q^{\prime}$ controlling the dissipation of 

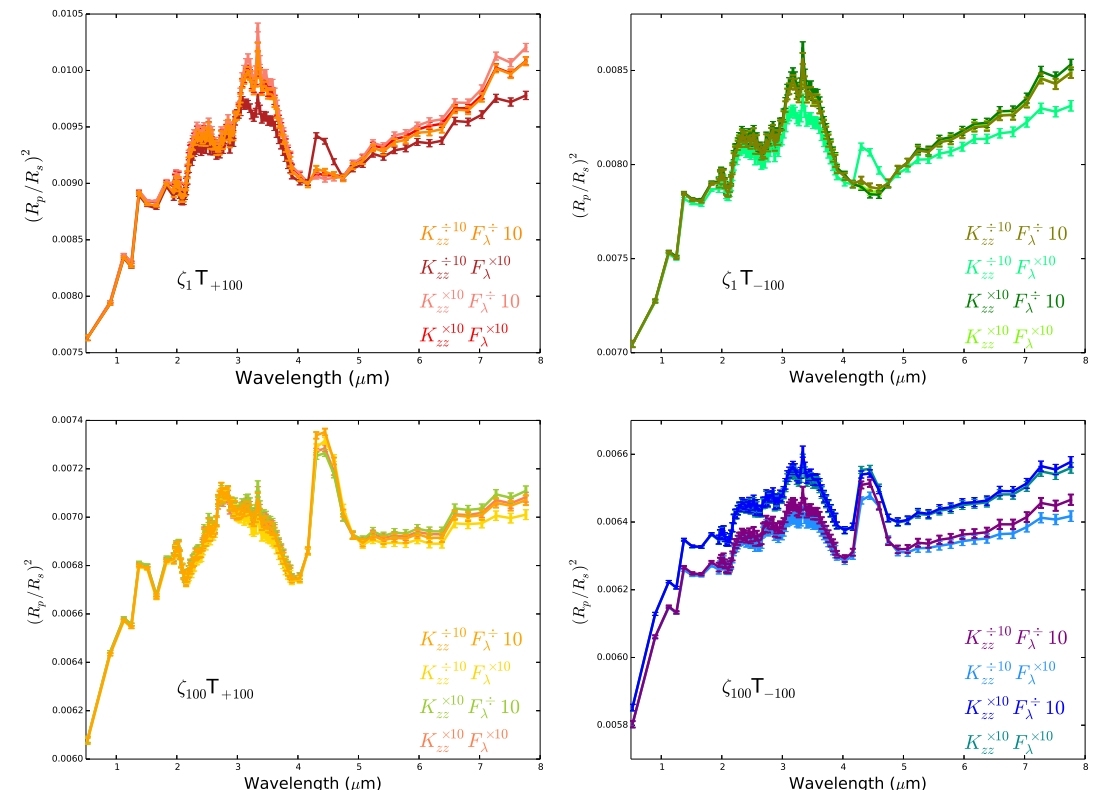

Fig. 4 Synthetic transmission spectra from 16 models of GJ $3470 \mathrm{~b}$ as they will be observed by ARIEL. The resolutions are 10,100, and 30, according to the spectral range, as explained in Table 1 The error bars correspond to a SNR of 20. The meaning of the different colors is explained on each plot.

tidal forces. $Q^{\prime}$ verifies the relation $k_{2} \Delta t=3 /\left(2 Q^{\prime} n\right)$, where $k_{2}$ is the Love number of degree 2 (Goldreich and Soter 1966), $\Delta t$ is the time lag (Hut 1981), and $n$ is the orbital mean motion (Leconte et al 2010). To estimate $Q^{\prime}$, they compared the age of GJ 436 system with the circularisation timescale $\tau_{e}=e / \dot{e}$ and found that $Q^{\prime}$ was greater than $10^{5}$, given the observed eccentricity. This limit value of $Q^{\prime}$ leads to an internal temperature $T_{\text {int }}<560 \mathrm{~K}$ (see Fig. 1 of Agúndez et al 2014a). For a detailed discussion on the tidal heating in exoplanet atmospheres, we refer the reader to Leconte et al (2010).

Agúndez et al (2014a) considered four internal temperatures that satisfy the above criterion: 100, 240, 400, and 560 K. In Agúndez et al (2014a), different metallicities were investigated, but here we will present only one case (i.e. $10 \times$ solar metallicity) and focus on the effect of different internal heating. The eddy diffusion coefficient is the same for each model and has been estimated from the GCM model of Lewis et al (2010) (i.e. $\mathrm{K}_{z z}$ varying between $10^{8}$ and $10^{11} \mathrm{~cm}^{2} \mathrm{~s}^{-1}$ depending on the pressure level.)

As can be seen in Fig. 5, the various internal temperatures lead to different temperature structures, particularly in the deep atmosphere, with subsequent consequences for the chemistry. Each thermal profile crosses the $\mathrm{CO}=\mathrm{CH}_{4}$ equilibrium line at different pressure levels, that will lead to differences in the steady-state abundances. For example, if quenching happens around 10 bar, for each profile, the quenching level is located on one side or the other of this equilibrium line. Thus, because of quenching, the chemical composition of the atmosphere will be 
different. As one can see in Fig. 6, the higher the internal temperature, the higher (lower) the abundance of carbon monoxide (methane) in the deep atmosphere. These variations of abundances lead to important changes regarding what is expected from thermochemical equilibrium: for all the thermal profiles with a $10 \times$ solar metallicity, $\mathrm{CO}$ is the major carbon-bearing species, instead of $\mathrm{CH}_{4}$. For the hottest profile, $\mathrm{CO}$ becomes in addition the major oxygen bearing-species instead of water thanks to vertical mixing.

These various atmospheric compositions produce different transmission spectra (Fig. 7). To calculate the spectra, we fixed the 10 bar pressure level to the planetary radius, which we set to $0.36 \mathrm{R}_{J u p}$, taking into account the possible error on the value given by Southworth (2010) (i.e. $0.37 \pm 0.018)$. A higher radius would produce the same spectral features but with larger transit depths, more discrepant from current observational data. A complete description of the spectra and the different molecular features can be found in Agúndez et al (2014b). We focus here on the SNR necessary to separate the different spectra. One can see on Fig. 7 that the atmosphere with the higher internal temperature is quite well separated from the other ones (between 0.8 and $230 \mathrm{ppm}$ ). Observations with a SNR of 10 are enough to differentiate it from the three other ones, which can be obtained combining four transits of GJ $436 \mathrm{~b}$ (see Table 2). In the spectral ranges between [2.2 - 2.4] and $[3.3-3.9] \mu \mathrm{m}$ the four spectra are very close to each other and are therefore not differentiable, even with a very good SNR. The absorption at these ranges are due to $\mathrm{CH}_{4}$. The spectrum corresponding to the model with an internal temperature of $400 \mathrm{~K}$ is closer to the two coldest ones with departures lower than $80 \mathrm{ppm}$. Thus, to differentiate the $400 \mathrm{~K}$ spectrum from the two other ones a SNR of 30 is

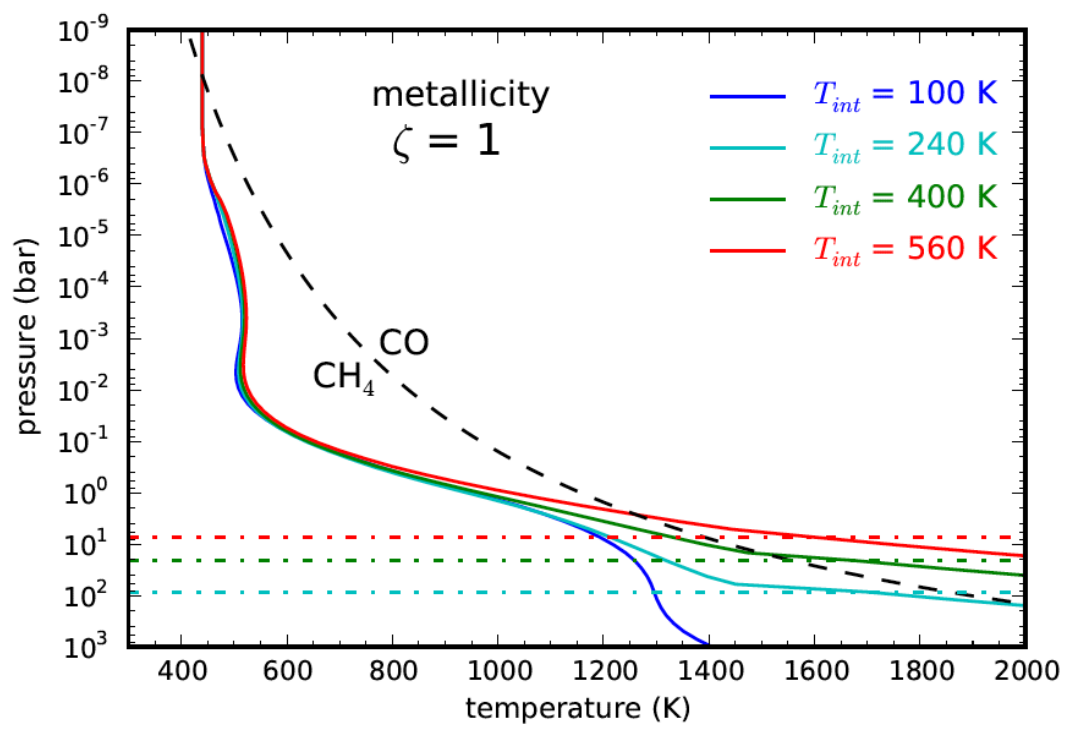

Fig. 5 Pressure-temperature profiles (full lines) of GJ 436b assuming different internal temperatures: $100 \mathrm{~K}$ (dark blue), $240 \mathrm{~K}$ (cyan), 400K (green), and $560 \mathrm{~K}$ (red). The dashed-dotted lines represent the transition between the radiative and the convective zone. The black dashed line represent the equilibrium line $\mathrm{CO} / \mathrm{CH}_{4}$. From Agúndez et al (2014b). 
required. Only the features around 1.4 and $4.5 \mu \mathrm{m}$ can be separated with a lower SNR (10 or 20). To be able to draw high-confidence conclusions, a higher SNR is advisable. Finally, the spectra corresponding to the two coldest thermal profiles are too similar and not differentiable whatever the SNR of the acquisitions.

\subsection{Elemental carbon/oxygen ratio}

Elemental abundances can have a crucial effect on the atmospheric composition of exoplanets. Several studies have been undertaken on this subject such as Line et al (2010); Madhusudhan et al (2011); Madhusudhan (2012); Kopparapu et al (2012); Moses et al (2013); Venot et al (2015); Mollière et al (2015); Heng and Lyons (2016); Rocchetto et al (2016) in which they also studied the consequences of different $\mathrm{C} / \mathrm{O}$ ratios on the observations. Most of these studies focused on hot Jupiters and revealed that as the $\mathrm{C} / \mathrm{O}$ ratio increases, abundances of hydrocarbons species increase also. Venot et al (2015) studied less hot exoplanets and found that for atmospheres with a temperature around $500 \mathrm{~K}$, changing the $\mathrm{C} / \mathrm{O}$ ratio
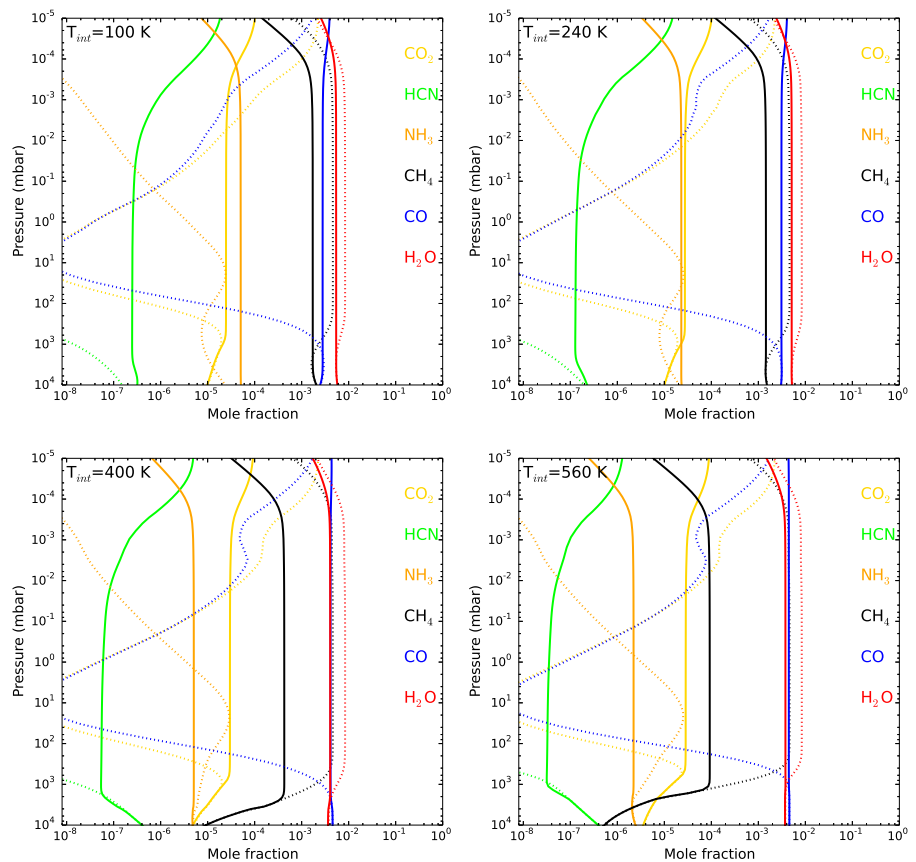

Fig. 6 Chemical composition of GJ $436 \mathrm{~b}$ with a metallicity of $10 \times$ solar metallicity and different internal temperature $(100 \mathrm{~K}, 240 \mathrm{~K}, 400 \mathrm{~K}$, and $560 \mathrm{~K})$ as labeled on each panel. Steady-state composition (full lines) is compared to thermochemical equilibrium (dashed lines). Adapted from Agúndez et al (2014b). 
from solar $(\mathrm{C} / \mathrm{O}=0.54)$ to twice solar $(\mathrm{C} / \mathrm{O}=1.1)$ has only a minor effect on the chemical composition and on the synthetic spectra (see Fig. 8). These two synthetic spectra would be very difficult to differentiate, even with a very good SNR.

Indeed, at this temperature, thermochemical equilibrium predicts that the most abundant heavy species remain the same at $\mathrm{C} / \mathrm{O}$ solar or above 1, i.e. $\mathrm{H}_{2} \mathrm{O}$ and $\mathrm{CH}_{4}$ (Fig. 9). Whereas the abundance of $\mathrm{H}_{2} \mathrm{O}$ does not change, $\mathrm{CH}_{4}$ sees its abundance doubling and becoming slightly above that of water. For both $\mathrm{C} / \mathrm{O}$ ratios, at $500 \mathrm{~K}, \mathrm{CO}$ has a very low abundance $\left(\sim 10^{-17}\right.$ at 1 bar $)$. Consequently, as the main species (after $\mathrm{H}_{2}$ and $\mathrm{He}$ ) are $\mathrm{H}_{2} \mathrm{O}$ and $\mathrm{CH}_{4}$ whatever the $\mathrm{C} / \mathrm{O}$ ratio at this temperature, chemical kinetics is governed by the same reactions, leading to similar atmospheric composition.

In agreement with previous studies aforementioned, Venot et al (2015) found that when dealing with hotter planets, the effect of the elemental abundances is much more important. The increase of the $\mathrm{C} / \mathrm{O}$ ratio leads to an important increase (by several orders of magnitude) in the abundance of hydrocarbon species (i.e. $\mathrm{CH}_{4}, \mathrm{C}_{2} \mathrm{H}_{2}$, etc.), accompanied with a decrease of the abundance of water (see Fig. 10). Here again, thermochemical equilibrium calculations provide an explanation for these results. Figure 9 shows that at $\mathrm{T}=1500 \mathrm{~K}$, contrary to $\mathrm{T} \lesssim 1000 \mathrm{~K}$, the major species change with the $\mathrm{C} / \mathrm{O}$ ratio. For a solar value, $\mathrm{H}_{2} \mathrm{O}$ and $\mathrm{CO}$ are the main heavy species and $\mathrm{CH}_{4}$ is less abundant by more than two orders of magnitude. On the contrary, for a $\mathrm{C} / \mathrm{O}$ ratio above 1 , the abundance of water is much lower and the major species are $\mathrm{CO}$ and $\mathrm{CH}_{4}$. Consequently, the main

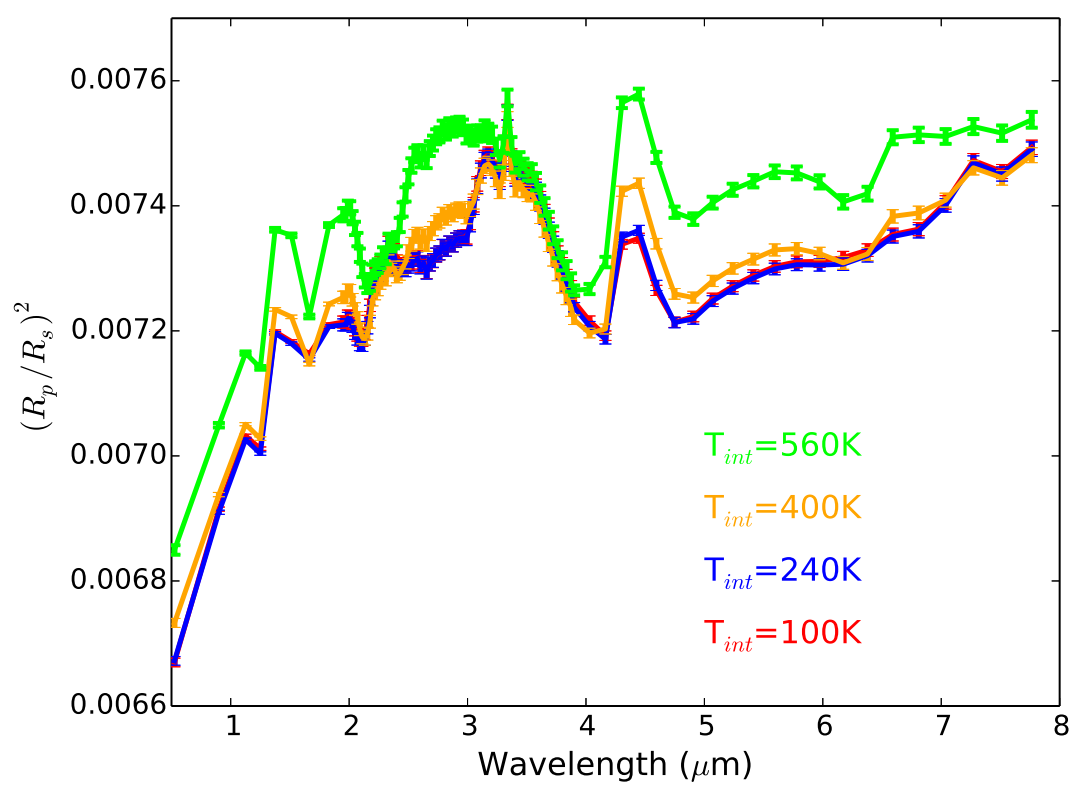

Fig. 7 Synthetic transmission spectra of GJ 436b with a high metallicity $(10 \times$ solar $)$ and different internal temperature (100 K, $240 \mathrm{~K}, 400 \mathrm{~K}$, and $560 \mathrm{~K})$. Spectra are binned to ARIEL resolution, as explained in Table 1 The error bars correspond to a SNR of 30. 

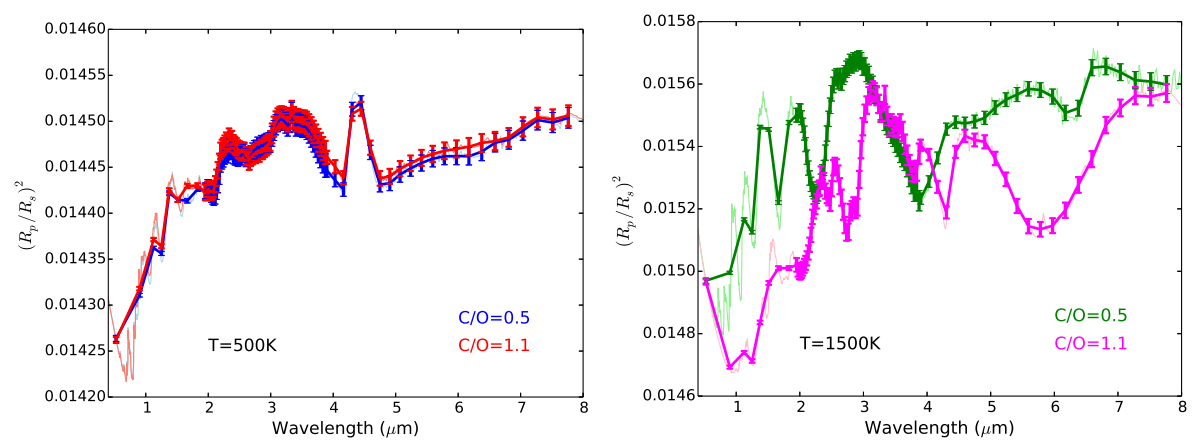

Fig. 8 Synthetic transmission spectra for an exoplanetary atmosphere with a temperature around $500 \mathrm{~K}$ (left) and $1500 \mathrm{~K}$ (right). Two $\mathrm{C} / \mathrm{O}$ ratios are represented: 0.5 (blue/green) and 1.1 (red/magenta). We assumed the test-planet was orbiting a Sun-like star and took the SNR of HD 189733b. The bold and low-resolution spectra are simulated ARIEL observations. The resolutions are 10,100, and 30, according to the spectral range, as explained in Table 1 The error bars correspond to a SNR of 20. The fainter curves are the corresponding higher-resolution spectra $(\mathrm{R}=300)$.

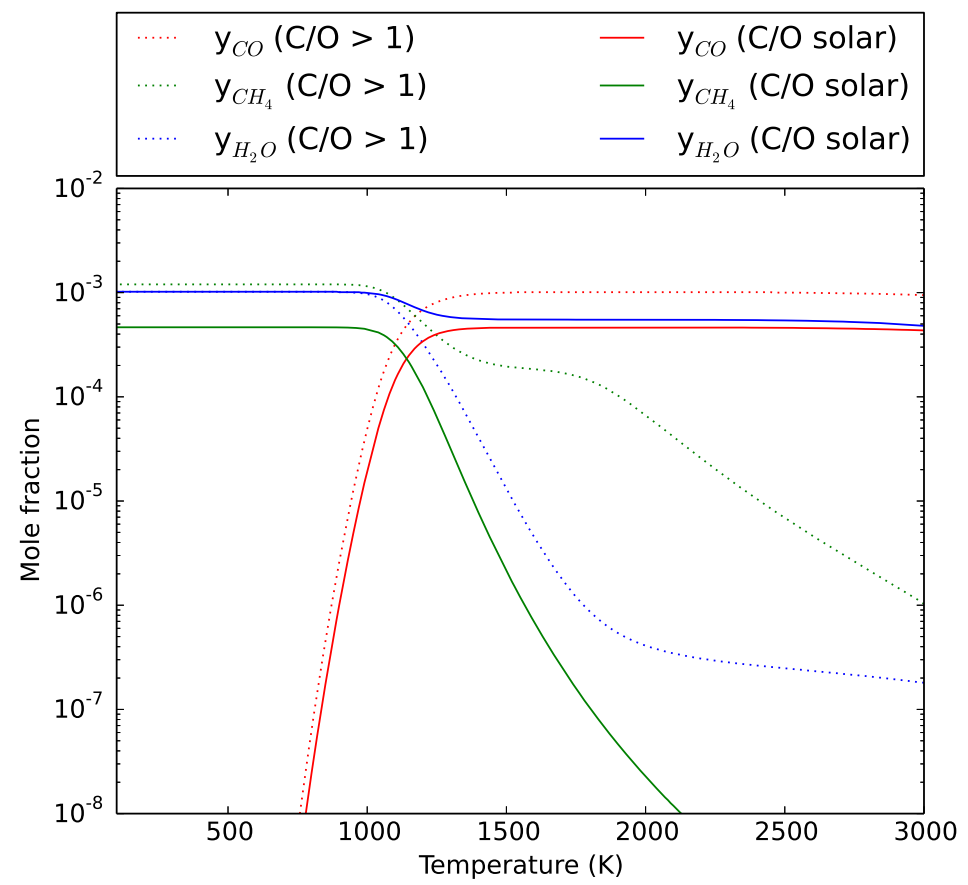

Fig. 9 Abundances of $\mathrm{CO}$ (red), $\mathrm{CH}_{4}$ (green), and $\mathrm{H}_{2} \mathrm{O}$ (blue) at thermochemical equilibrium as a function of temperature. Calculations have been done for $\mathrm{P}=1$ bar and for two Carbon/Oxygen ratio: solar (full lines) and $>1$ (dotted lines).

reactions and destruction/formation pathways in a hot atmosphere will be different 


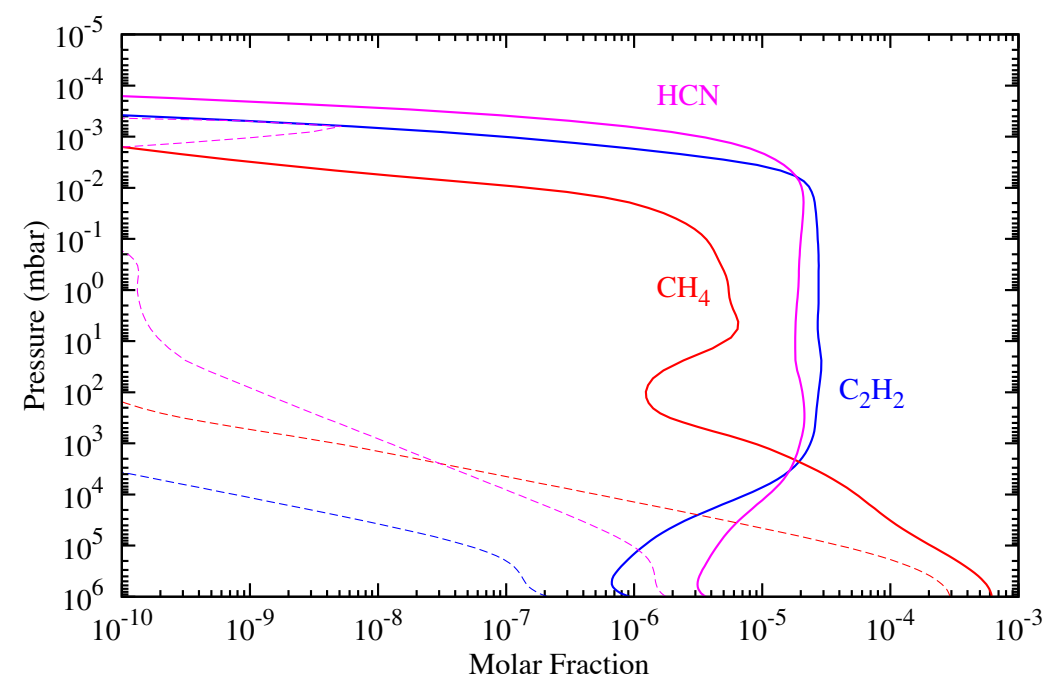

Fig. 10 Vertical abundance profiles of $\mathrm{CH}_{4}$ (red), $\mathrm{C}_{2} \mathrm{H}_{2}$ (blue), and $\mathrm{HCN}$ (pink) for an atmosphere with a temperature around $1500 \mathrm{~K}$ assuming two different $\mathrm{C} / \mathrm{O}$ ratios: solar (dashed lines) and twice solar (full lines). Adapted from Venot et al (2015), reproduced with permission (c) ESO.

depending on the $\mathrm{C} / \mathrm{O}$ ratio. See also Moses et al $(2013)$ for a detailed study on the effect of the $\mathrm{C} / \mathrm{O}$ ratio on hot exoplanetary atmospheres.

This difference of chemical composition is highly visible on the synthetic spectra. As one can see in Fig. 8, the two transmission spectra corresponding to the $\mathrm{C}$-rich and the $\mathrm{C} / \mathrm{O}$ solar cases are very separate and can be differentiated easily with a low SNR (5). The shape of the spectrum corresponding to the $\mathrm{C} / \mathrm{O}$ solar case is mainly due to water absorption, with some features of $\mathrm{CO}$ and $\mathrm{CO}_{2}$ around $4.8 \mu \mathrm{m}$. In contrast, the C-rich spectrum owes its form to $\mathrm{CH}_{4}, \mathrm{CO}, \mathrm{HCN}$, and $\mathrm{C}_{2} \mathrm{H}_{2}$. Because of their strong spectral features around $14 \mu \mathrm{m}$, these latter two absorbers can be used as tracers for the C/O ratio in warm exoplanet's atmosphere (i.e. Kopparapu et al 2012; Venot et al 2015). This technique has been used by Tsiaras et al (2016) to suggest that the super-Earth 55 Cancri e possesses a C-rich atmosphere, thanks to the detection of high amount of HCN.

As it has been shown by Rocchetto et al (2016), differentiating an atmosphere with a $\mathrm{C} / \mathrm{O}<1$ from a $\mathrm{C} / \mathrm{O}>1$ is quite straightforward with a retrieval process as the spectra are really dissimilar but it might be more difficult to retrieve a more precise value of the $\mathrm{C} / \mathrm{O}$ ratio. Indeed, an atmosphere with a $\mathrm{C} / \mathrm{O}=0.5$ has a spectrum very close to that of an atmosphere with a $\mathrm{C} / \mathrm{O}=0.6$ for instance. 
5 Influence of stellar parameters

5.1 Spectral type of stars

A crucial factor for understanding exoplanet atmospheres is the host star. Stellar irradiation largely determines the temperature of the atmosphere and, in addition, the stellar flux is observable in the spectra through reflected and transmitted light. Furthermore, UV flux irradiation drives photochemistry in the upper atmospheres of exoplanets.

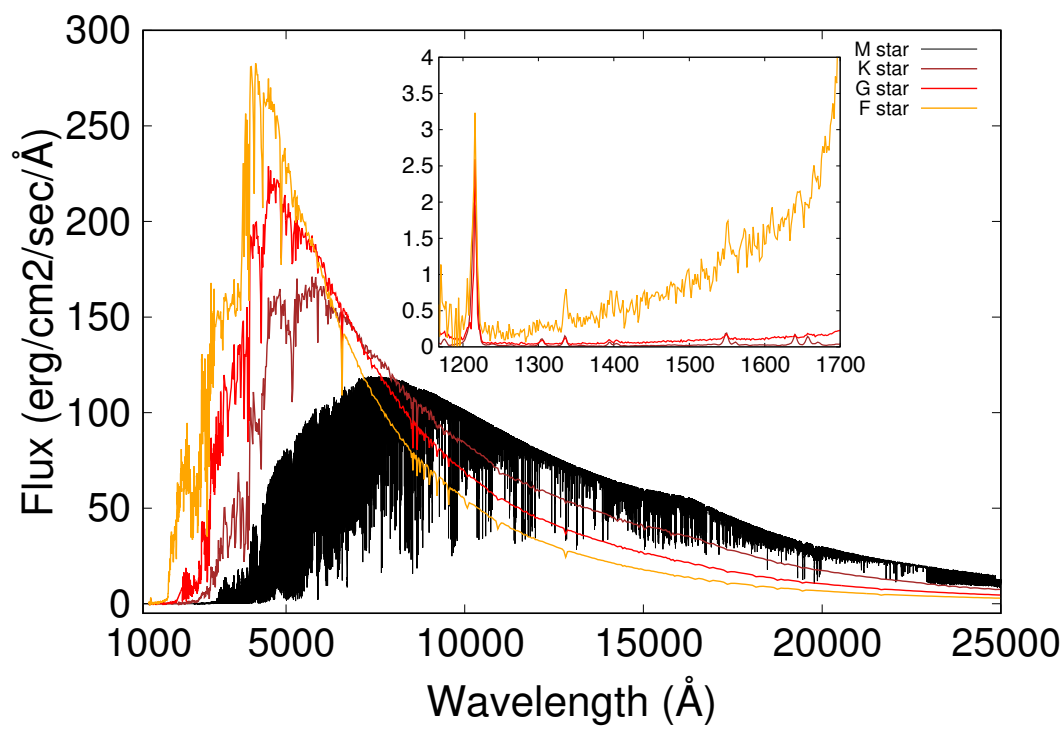

Fig. 11 Stellar flux at $1 \mathrm{AU}$ as a function of wavelength of an M (black), K (brown), G (red) and $\mathrm{F}$ (orange) star. FarUV flux can be seen in the zoomed region.

Fig. 11 shows the stellar spectra of an M, K, G and F star. These spectra are a combination of data from the ATLAS synthetic spectra (Kurucz 1979) and UV observations from the International Ultraviolet Explorer (IUE) as explained in Rugheimer et al (2013). For the M star, we are showing the emission flux of an inactive M star from a model by Allard et al (2001). As seen in the figure, stars of different stellar types emit very different fluxes, creating a different response in the atmospheres of the planets around them.

Miguel and Kaltenegger (2014) studied the effect of stellar flux on the chemistry of extrasolar giant planets and mini-Neptunes. They studied the change in atmospheric chemical composition for planets around different stars and semi-major axis ranging from 0.01 to 0.1 AU. In Fig. 12 we show how the atmospheric composition of an exoplanet is affected when the planet is exposed to irradiation from different stellar types stars. The simulations were performed for a planet located a $0.025 \mathrm{AU}$ and exposed to the irradiation of the stars shown in Fig. 11. The TP 
profiles for each planet were calculated using a gray atmosphere (Guillot 2010), as explained in Miguel and Kaltenegger (2014). The results show that the stellar flux (especially in the UV domain) strongly influences the photochemistry, especially the photolysis of $\mathrm{H}_{2} \mathrm{O}$. Since water drives the chemistry in the region between $10^{-4}$ and $10^{-6}$ bars of these planet's atmospheres, the change in water affects the chemistry of the other major species. A planet around an F star receives a much higher amount of UV flux than a planet around an M star, therefore photolysis of water is much more efficient for the planets around F and G stars than it is on planets around cool $\mathrm{K}$ and $\mathrm{M}$ stars. When looking at pressures around $10^{-4}$ bars, we see that the change in $\mathrm{H}_{2} \mathrm{O}$ and $\mathrm{CO}_{2}$ between the two extreme cases (planet around $\mathrm{M}$ and $\mathrm{F}$ stars) is of approximately two orders of magnitude, and the change in composition of $\mathrm{CH}_{4}$ and $\mathrm{H}$ is substantial. The variation of $\mathrm{CH}_{4}$ abundance between the two extreme values at that pressure is of approximately 10 orders of magnitude and the variation in $\mathrm{H}$ mixing ratio is of 5 orders of magnitude.

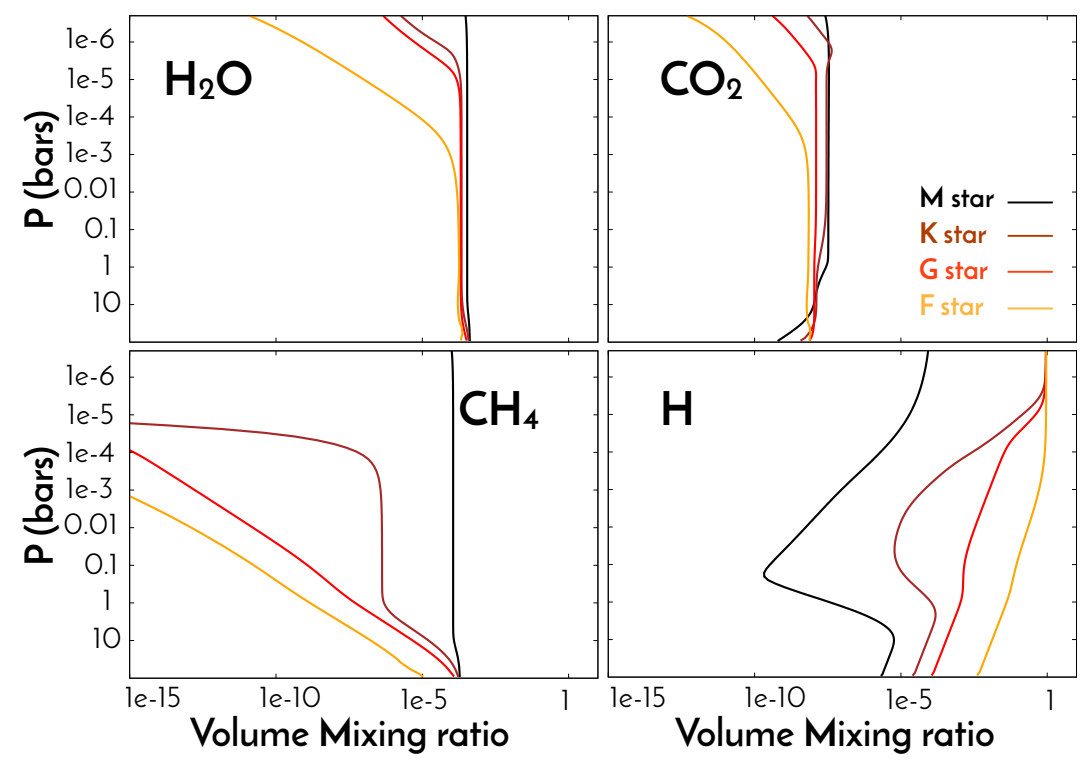

Fig. 12 Vertical chemical abundances profiles of $\mathrm{H}_{2} \mathrm{O}, \mathrm{CO}_{2}, \mathrm{CH}_{4}$, and $\mathrm{H}$ in an exoplanet atmosphere when the planet orbits different stellar type stars. The different stellar types (F, $\mathrm{G}, \mathrm{K}, \mathrm{M})$ are represented by different colors.

We calculated the transmission spectra for the four models presented in Fig. 12. The four spectra present very different transit depth because the F, G, K, and $\mathrm{M}$ stars have different radii $\left(1.5 R_{\odot}, 1.1 R_{\odot}, 0.8 R_{\odot}\right.$, and $0.62 R_{\odot}$ respectively) while the planet keeps the same properties (i.e. $R=R_{\text {Jup }}$ and $M=0.969 M_{\text {Jup }}$ ). Apart from this obvious difference, we notice that the spectra for the F, G, and $\mathrm{K}$ host stars present a similar shape. All the absorption is due to water, except the absorption feature around $4.8 \mu \mathrm{m}$, which is the contribution of $\mathrm{CO}_{2}$ and $\mathrm{CO}$. The spectrum of the planet orbiting a $\mathrm{M}$ star presents all the absorption features of $\mathrm{H}_{2} \mathrm{O}$ (i.e. around $0.95,1.15,1.4,1.9$, and $6.7 \mu \mathrm{m}$ and on the ranges $[2.5-3]$ and 

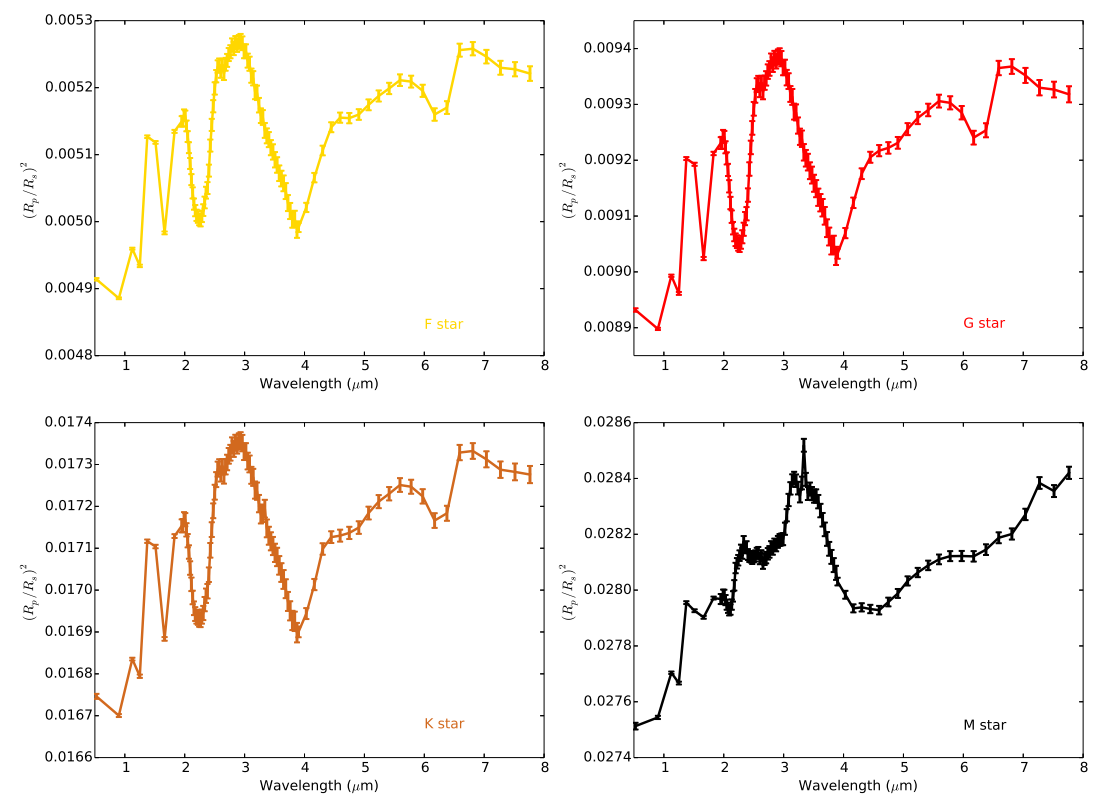

Fig. 13 Synthetic transmission spectra of a Jupiter-like planet orbiting around an F, G, K, and $\mathrm{M}$ star as it will be observed by ARIEL. The resolutions are 10, 100, and 30, according to the spectral range, as explained in Table 1 The error bars correspond to a SNR of 30.

[4.2-5.8] $\mu \mathrm{m})$ to which are superimposed those of $\mathrm{CH}_{4}$ around 1.6 and $2.3 \mu \mathrm{m}$ and on the ranges [3-4] and [7-8] $\mu \mathrm{m}$. The absorption features of these two molecules will be easily characterized by ARIEL with observations with a $\mathrm{SNR} \geq 15$.

The effect of stellar flux is extremely important for hot and warm exoplanet's atmospheres, but it can also affect smaller planets at wider orbits. The main effect of stellar irradiation in rocky planet's atmospheres located in the habitable zone (Kopparapu et al 2014) is that as temperature increases (going from cold K stars to hot $\mathrm{F}$ stars) the atmospheres show more $\mathrm{O}_{3}$, more $\mathrm{OH}$, less tropospheric $\mathrm{H}_{2} \mathrm{O}$ (but more stratospheric $\mathrm{H}_{2} \mathrm{O}$ ), and less stratospheric $\mathrm{CH}_{4}, \mathrm{~N}_{2} \mathrm{O}$, and $\mathrm{CH}_{3} \mathrm{Cl}$, showing that the effect of the star is extremely important for the modelling and thus the understanding of their atmospheres (Rugheimer et al 2013).

\subsection{Stellar activity}

M stars are a special case. These stars make up the vast majority of stars in the Galaxy and we expect to find many more planets around M stars in the near future. The future telescope ARIEL will allow us to characterize their atmospheres, so it is important to anticipate their possible compositions. M stars are cool stars, but not very quiet: they present a high photospheric activity that causes an excess in the UV flux produced when comparing to the black body radiation. They can also present flares that might affect the chemistry in their atmospheres, as explained in Section 5.2 .2 . 

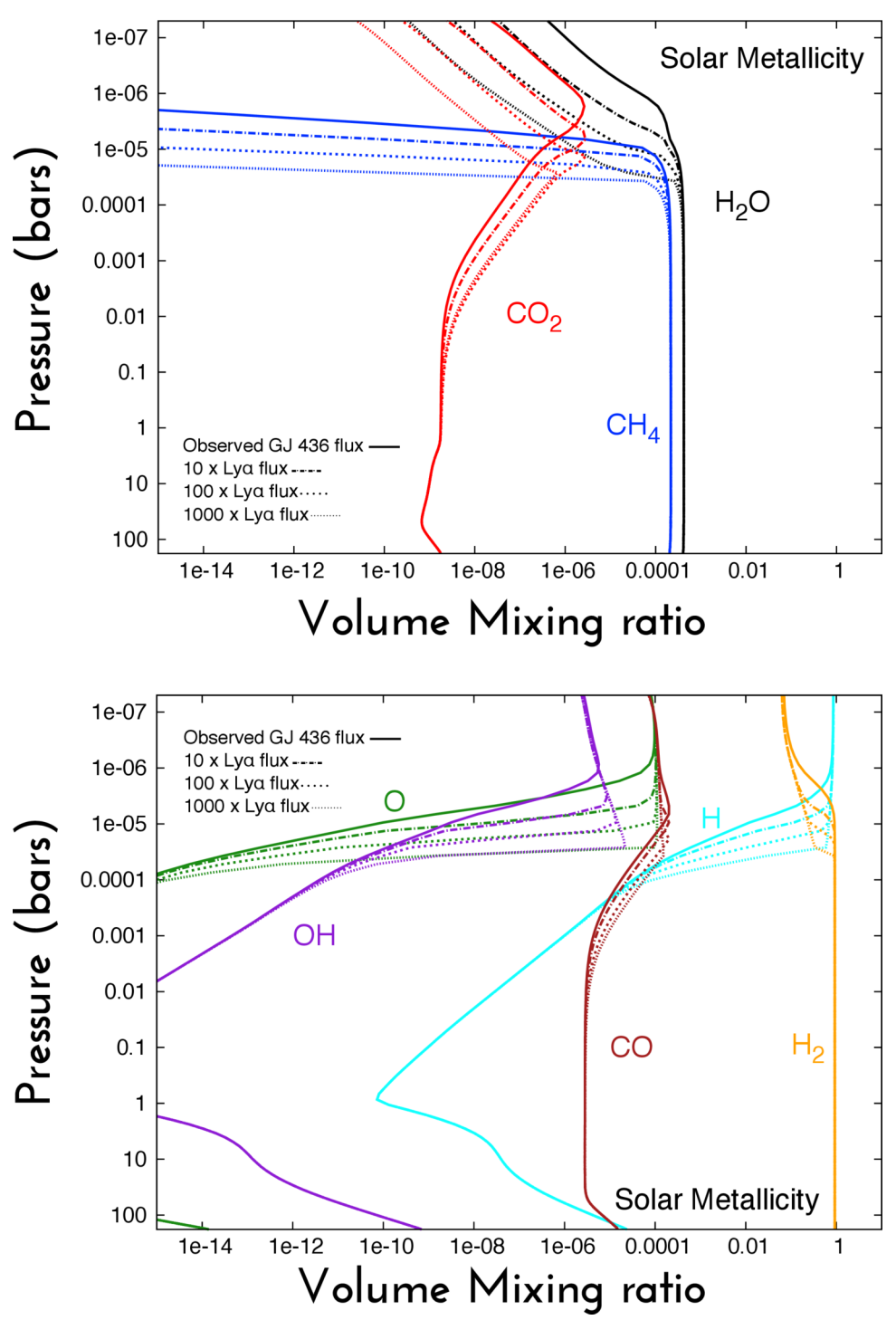

Fig. 14 Effect of UV flux emitted by M stars (specifically Lyman $\alpha$ radiation) on an exoplanet atmosphere. In this case the planet used as an example is the mini-Neptune GJ 436b. The figure shows the results of adopting different levels of Lyman $\alpha$ flux on the abundance of key species in its atmosphere when using a solar composition. Figure adapted from Miguel et al (2015).

\subsubsection{Lyman $\alpha$}

The brightest emission line in the UV of $\mathrm{M}$ stars is the Lyman $\alpha$ radiation at 1216.67 Ang, with a percentage of total UV flux from the star in the Lyman $\alpha$ line between 37 and 75 per cent compared to 0.04 per cent for the Sun (France et al 2013). A number of M stars were observed in the UV thanks to the efforts of the 
MUSCLES team (France et al 2016), and the Lyman $\alpha$ lines of those stars were reconstructed (Youngblood et al 2016).

Miguel et al (2015) studied the effect of incoming Lyman $\alpha$ radiation on the photochemistry of mini-Neptunes' atmospheres made of solar and higher metallicities. They studied the effect of a star with different levels of Lyman $\alpha$ flux and its effect on the chemistry. In the solar metallicity atmosphere, their results show that $\mathrm{H}_{2} \mathrm{O}$ presents the largest change as it absorbs most of the radiation, shielding other molecules. It can be seen on Fig. 14 that $\mathrm{H}_{2} \mathrm{O}$ dissociates very efficiently and the products of that dissociation $(\mathrm{O}$ and $\mathrm{OH})$ affect the chemistry of other species (Moses et al 2011). For higher metallicities, $\mathrm{CO}_{2}$ is also highly effected.

We calculated the synthetic transmission spectra corresponding to these different cases and binned them to ARIEL resolution (Fig. 15). We observe variations around $2.5 \mu \mathrm{m}$ and $3.5 \mu \mathrm{m}$ and on the range [7-8] $\mu \mathrm{m}$, which are due to changes in $\mathrm{CH}_{4}$ abundance whereas the variations around $4.3 \mu \mathrm{m}$ correspond to the increase of $\mathrm{CO}_{2}$ abundance with metallicity. These spectral variations are rather small but could be detected at some wavelength, with a very good SNR. In the case of GJ $436 \mathrm{~b}$, a SNR $\geq 30$ is necessary. These results further show that we need to obtain good observations of the stellar fluxes, especially in the UV to get a proper interpretation of the planetary spectra.

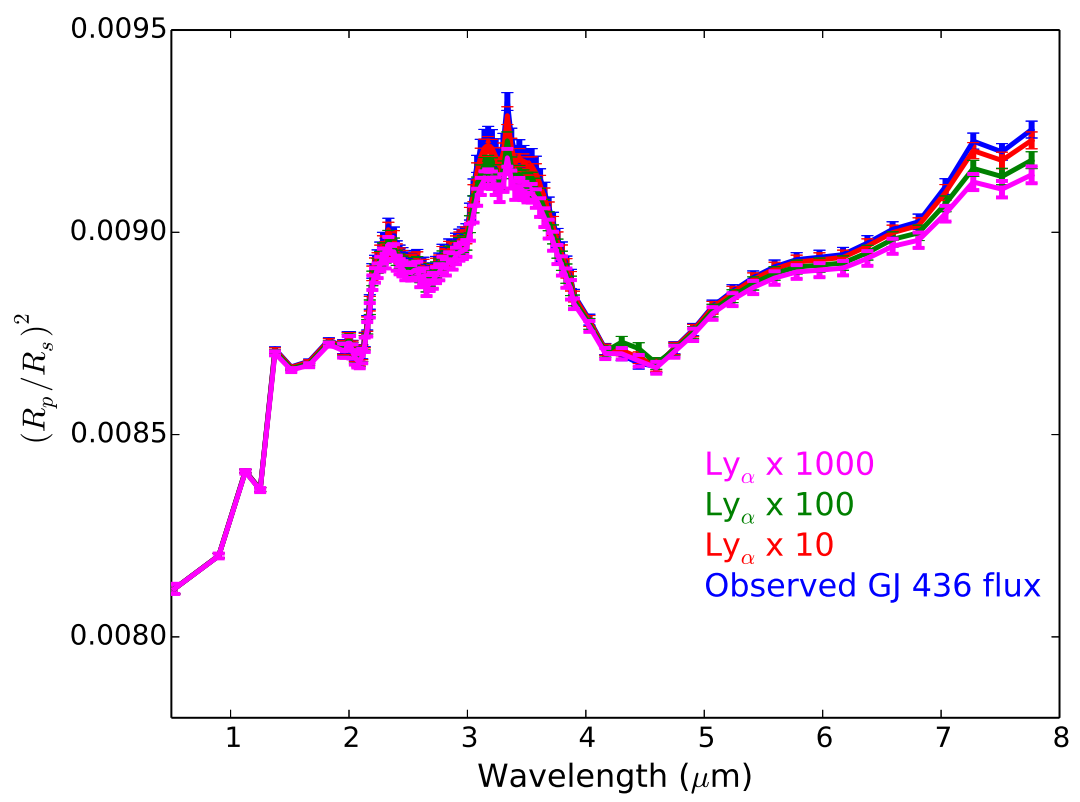

Fig. 15 Synthetic transmission spectra of the mini-Neptune GJ 436b when receiving different levels of Lyman $\alpha$ flux. Spectra are binned to ARIEL resolution, as explained in Table 1 The error bars correspond to a SNR of 30 . 


\subsubsection{Flares}

Active stars, in particular M stars, are subject to stellar variations, which may have an impact on the chemical composition of exoplanets. Venot et al (2016) studied to what extent a stellar flare can influence the atmospheric composition of hot/warm exoplanets and the resulting spectra. They found that the increase of UV flux associated to a single flare event from an $\mathrm{M}$ star can modify the abundances of the main species $\left(\mathrm{H}, \mathrm{NH}_{3}, \mathrm{CO}_{2}\right.$, etc. $)$ by several orders of magnitude and down to a pressure level of $\sim 1$ bar (Fig. 16).

However, a unique flare event is not realistic, as an active star undergoes flare several time in its lifetime. To model a more realistic case, they simulated a series of flares occurring periodically (every five hours) and found that the chemical abundances of species oscillate around a mean value that evolves with time towards a limiting value (Fig. 17). The number of flare events required to reach a limiting value depends on the species and on pressure. Here, the flare is happening with the same intensity and comes back with the same period. The mean and limiting values might be more difficult to reach in reality, with flares of different intensity happening with inconstant period. The conclusion that can be drawn from this study is that planets around very active stars (undergoing frequent flares) are probably never at a steady-state but are constantly and permanently altered by flare events. These important variations are detectable on the synthetic spectra as deviations from the steady-state can be up to 1200 ppm (Fig 18.

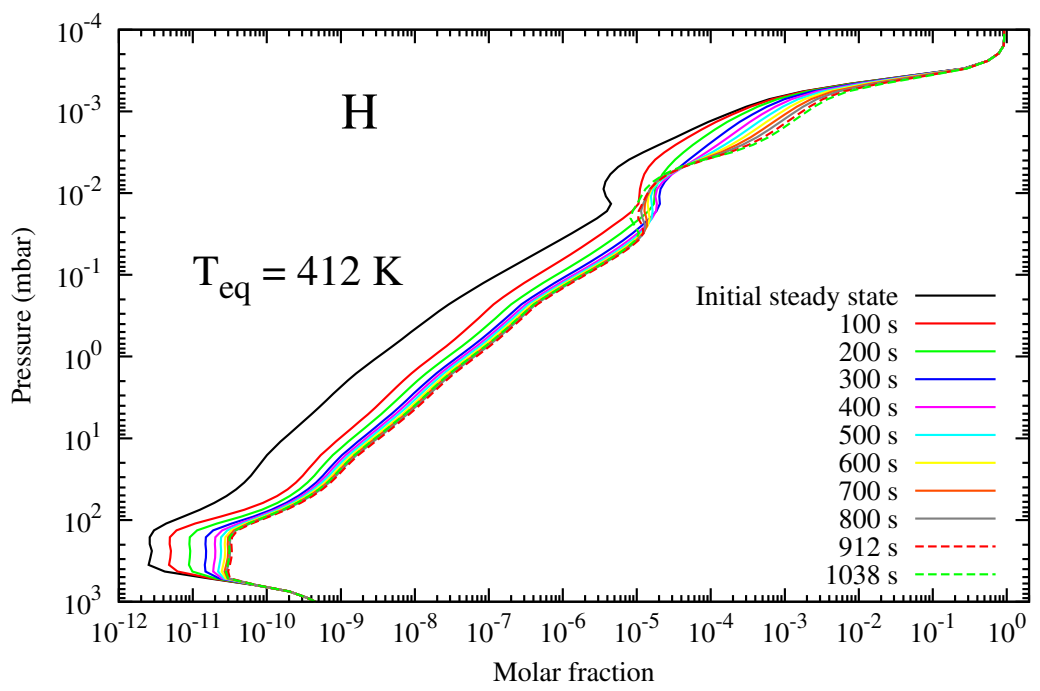

Fig. 16 Relative abundances of hydrogen during the first 1038 seconds of a flare event. The planet is a warm Neptune with an equilibrium temperature of 412 K. From Venot et al (2016). 


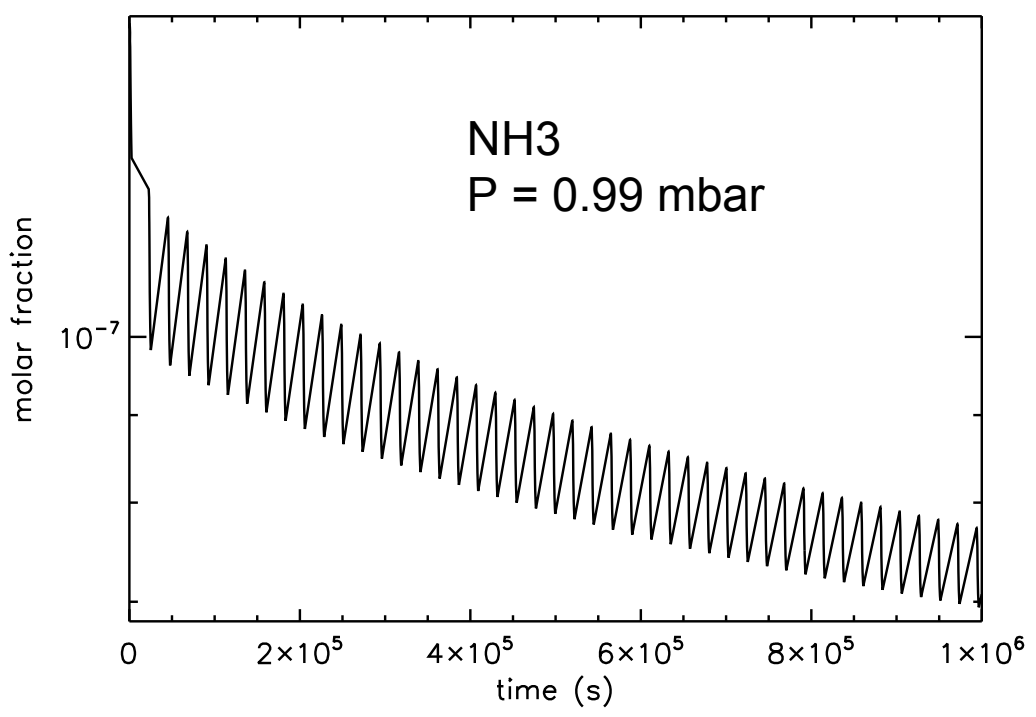

Fig. 17 Temporal evolution of the atmospheric abundance of ammonia in a planet orbiting a flaring star. The host star undergoes energetic flares every five hours. The planet is a warm Neptune with an equilibrium temperature of $1303 \mathrm{~K}$. From Venot et al (2016).

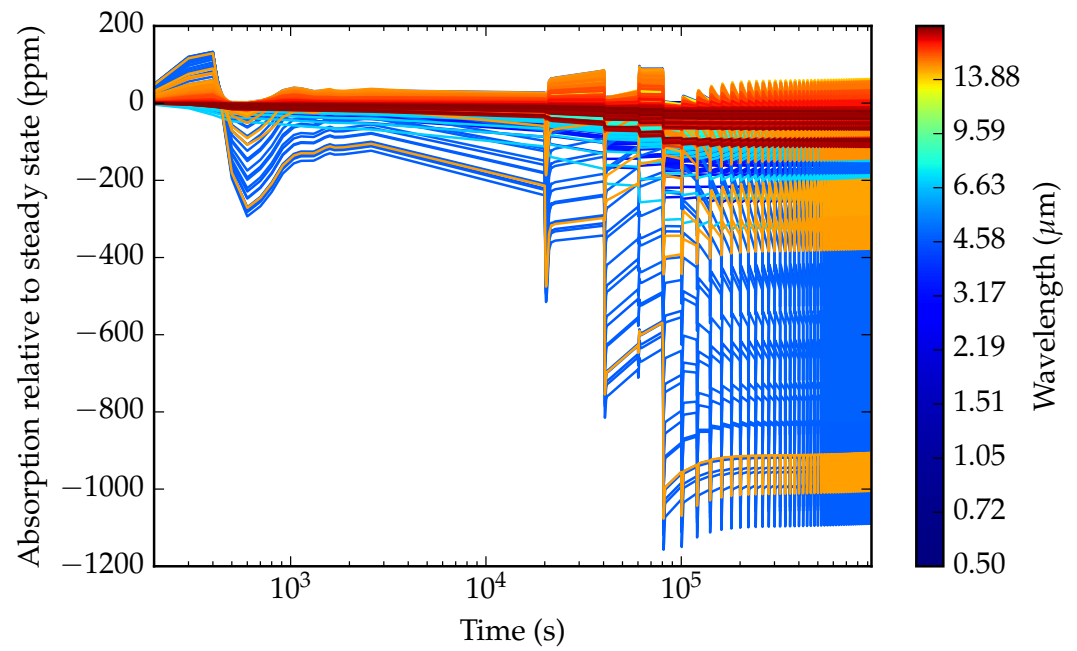

Fig. 18 Variations of the spectra as a function of time and wavelength (color bar) with respect to the steady-state when the star undergoes a flare every 5 hours. From Venot et al (2016). 


\section{Progress in terms of modelling}

Most chemical models of hot/warm exoplanets have involved one-dimensional vertical profile models with a specified input temperature profile. However, recent model developments have extended this to take into account 1) consistency of the chemistry with the temperature profile and 2) extend the model dimension to include the effects of horizontal transport.

\subsection{Coupling chemistry-thermal profile}

As was shown previously (Sect. 4), various model parameters can have a large impact on the chemical abundances. In particular, for a given elemental composition, the temperature profile largely determines calculated chemical abundances through the temperature-dependence of the rate constants. In addition, if vertical mixing is taken into consideration, the location of the quench point is a playoff between the chemical timescale and mixing timescale, which depend on the temperature and mixing strength $\left(\mathrm{K}_{z z}\right)$ respectively.

However, the temperature profile is in turn dependent on the chemical composition, as it controls the opacity and hence the absorption and emission of radiation. Drummond et al (2016) used a 1D radiative-convective equilibrium model, which includes a chemical kinetics scheme, using the chemical network of Venot et al (2012), to solve for the temperature profile which is consistent with nonequilibrium abundances (i.e. including vertical mixing and photochemistry). It was found that the process of vertical mixing can have a strong influence on the temperature profile compared with the temperature profile consistent with chemical equilibrium, depending on the strength of the mixing. Figure 19 shows the pressure-temperature profiles for a model of HD 189733b, with profiles consistent with both chemical equilibrium and non-equilibrium (vertical mixing and photochemistry). Depending on the strength of the mixing, non-equilibrium chemistry can have an important influence on the temperature profile; locally increasing the temperature by up to $100 \mathrm{~K}$.

This change in the temperature due to non-equilibrium abundances has a feed back on the abundances themselves, through the temperature dependent rate constants. In this case, this leads to larger $\mathrm{CH}_{4}$ abundances and smaller $\mathrm{CO}$ abundances, compared with the model where the temperature profile is held fixed. The impact of photochemistry on the temperature profile is very small, since photochemistry is only important at low pressures $\left(P<10^{-5}\right.$ bar $)$ where the atmosphere is optically thin.

Calculating non-equilibrium abundances consistently with the temperature structure also has an impact on the simulated observations. Figure 20 shows the emission and transmission spectra for the same model, comparing chemical equilibrium, non-consistent non-equilibrium and consistent non-equilibrium cases. The spectra have been binned to the resolution of ARIEL (see Table 11. Note these spectra have been recalculated (see Section 2.2) from the thermal and composition profiles presented in Drummond et al (2016).

The emission spectra of the three cases (equilibrium, consistent non-equilibrium and non-consistent non-equilibrium) are all different to each other. However, Fig. 20 shows that of the two non-equilibrium cases, the consistent model (blue line) 


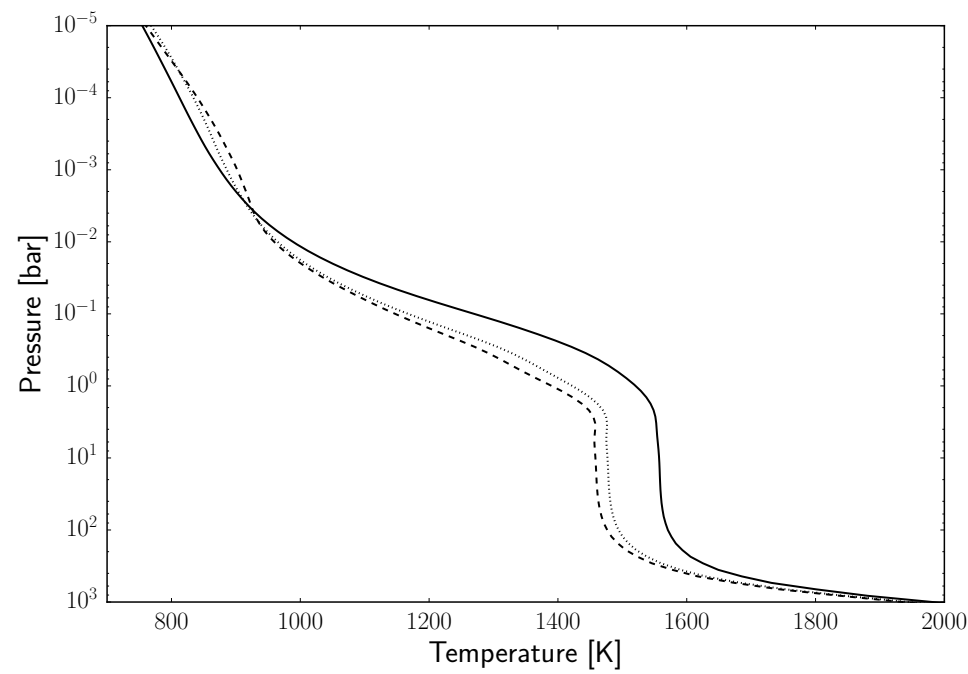

Fig. 19 The pressure-temperature profiles derived for HD 189733b assuming chemical equilibrium (dashed), and with vertical mixing and photochemistry included for $K_{z z}=10^{9} \mathrm{~cm}^{2} \mathrm{~s}^{-1}$ (dotted) and $K_{z z}=10^{11} \mathrm{~cm}^{2} \mathrm{~s}^{-1}$. From Drummond et al (2016), reproduced with permission (C)ESO.

is closer to the equilibrium model (green line) for most wavelength regions; there are however, exceptions to this, for example around $4.5 \mu \mathrm{m}$. This means that, depending on the wavelength, previous studies using a non-consistent approach could have overestimated the effect of non-equilibrium chemistry on the emission spectrum. In transmission the consistent and non-consistent non-equilibrium cases give broadly similar results.
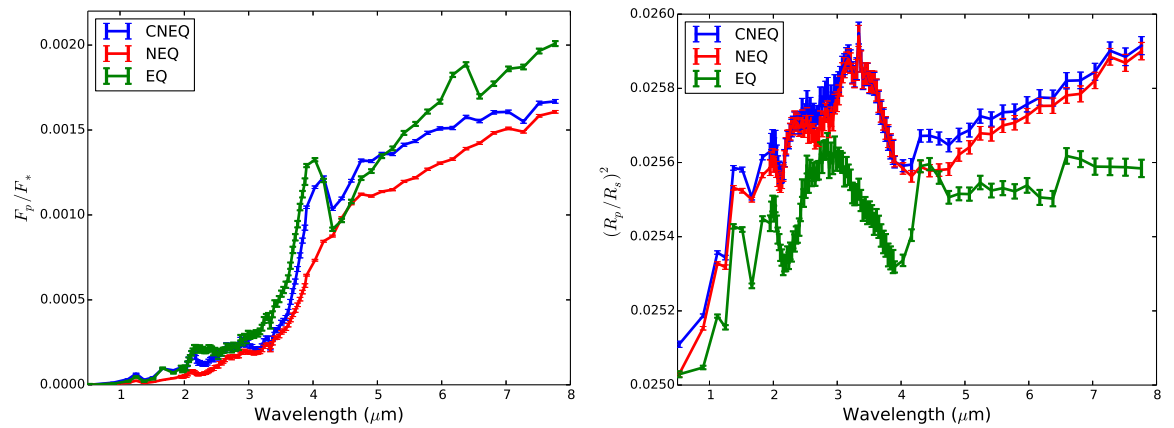

Fig. 20 The simulated emission (left) and transmission (right) spectra of HD 189733b that will be observed by ARIEL, for $K_{z z}=10^{11} \mathrm{~cm}^{2} \mathrm{~s}^{-1}$, showing the model assuming chemical equilibrium (EQ, green), the model with non-consistent non-equilibrium chemistry (NEQ, red) and the model with consistent non-equilibrium chemistry (CNEQ, blue). Spectra are binned to ARIEL resolution, as explained in Table 1 The error bars correspond to a SNR of 21. 
The difference between the consistent and non-consistent models can be understood in terms of energy balance of the atmosphere. The photosphere is the location in the atmosphere where the optical depth is approximately unity, and is where energy can freely escape to the top of the atmosphere. The pressure level of the photosphere is dependent on wavelength due to the wavelength-dependent opacity which, in turn, is determined by the chemical composition. The energy flux from the atmosphere, at a particular wavelength, is determined by the atmospheric temperature at the photospheric pressure level.

The main consequence of non-equilibrium chemistry, in this particular case, is to increase the abundances of $\mathrm{CH}_{4}$ and $\mathrm{NH}_{3}$ via vertical quenching. The corresponding increase in opacity shifts the photosphere to lower pressures, and lower temperatures, for the wavelengths at which these species absorb, changing the energy flux and the shape of the emission spectrum. While the spectral energy flux from the atmosphere may change, due to a changing composition, the total energy flux (i.e. integrated over wavelength) must balance the total energy input to the model atmosphere. Here, irradiation and internal heating are the only sources of energy to the atmosphere and are constant for all three cases.

Drummond et al (2016) found that the consistent non-equilibrium model gives the same integrated emitted energy flux as the equilibrium model, to within the expected accuracy of the calculations, as expected while the non-consistent nonequilibrium model shows a $39 \%$ decrease compared with the equilibrium model. The non-consistent model does maintain energy balance. To conserve energy balance the consistent calculation allows the temperature profile (in this case heats up) to evolve mitigate the effect of a shifting photosphere. We refer the reader to Drummond et al (2016) for a more detailed discussion.

The variations between these three models will be differentiable with ARIEL. With one single observation $(\mathrm{SNR}=7)$, the resolution of ARIEL is sufficient to separate the chemical equilibrium model from the two others, both in emission and transmission. Concerning the non-equilibrium chemistry models, separating the consistent one from the non-consistent one requires different SNR depending on the type of observation: for an observation during the secondary eclipse, a SNR of 7 allows to distinguish them for $\lambda>4 \mu \mathrm{m}$. In the range $2-4 \mu \mathrm{m}$, about nine observations are required, resulting in a SNR of 21 . On primary transit observations, the two models are more difficult to disentangle. For $\lambda<2 \mu \mathrm{m}$ and between 4 and $5 \mu \mathrm{m}$, the two spectra can be separated with a SNR of 14 , but elsewhere a SNR $>21$ is required. Note, however, that in the range $[2-2.5] \mu \mathrm{m}$ and $[3-4] \mu \mathrm{m}$, the two non-equilibrium chemistry models give very similar spectra that can't be distinguished, even with very high signal-to-noise ratios. Overall, this study shows that considering consistency between chemistry and temperature is very important in atmospheric modeling, as the variations on spectra between the different models are greater than the uncertainties on the future observations of ARIEL.

It is possible that studies not taking into account consistency of chemistry and temperature may have overestimated the impact of non-equilibrium chemistry on the spectrum, due to the models not conserving energy. An additional process not considered in Drummond et al (2016) is adjustments to the thermal profile due to energy changes associated with chemical reactions, which may also play a role in linking the chemical composition with the thermodynamics of the atmosphere. 


\subsection{Horizontal mixing}

All of the results presented so far have used a $1 \mathrm{D}$ code that approximates the atmosphere as a single column. However, short-period exoplanets are expected to be tidally locked, with large day-night temperature contrasts and fast horizontal winds. The effects of this three dimensional temperature structure and dynamics cannot be consistently captured with a 1D model. Coupling a complex chemical model to a 3D circulation model is a large computational challenge.

To date, two attempts have been made to include the effects of horizontal mixing in hot Jupiter atmospheres: 1) coupling a complex 3D model with a very simplified chemical scheme (Cooper and Showman 2006) and 2) coupling a complex chemical scheme with a simplified circulation model, a pseudo-2D model Agúndez et al 2012, 2014a). In this latter case, the 1D atmospheric column rotates as a solid body to mimic a uniform zonal wind; i.e. time is taken as an effective second spatial dimension.

In their model, Cooper and Showman (2006) include only $\mathrm{H}_{2} \mathrm{O}, \mathrm{CO}$, and $\mathrm{CH}_{4}$, which are the main carbon and oxygen bearing species. Their goal is to investigate the $\mathrm{CO} / \mathrm{CH}_{4}$ interconversion in the atmosphere of $\mathrm{HD} 209458 \mathrm{~b}$. They argue in terms of timescales estimates and find that vertical mixing is more important than horizontal mixing. They find that vertical quenching from a deep layer leads to a horizontally uniform composition for lower pressures. On the other hand, Agúndez et al (2014a) study the detailed chemical composition of HD 209458b and HD 189733b, with a chemical scheme involving more than 100 species. In both planets, they find that horizontal mixing is more important, and that the chemistry is quenched horizontally, with the chemistry of nightside being 'contaminated' with that of the dayside (see Fig. 21). Understanding the differences between the results of these two studies may require extending capabilities to consistently include a full chemical kinetics scheme in a 3D circulation model: a difficult, but important, challenge.
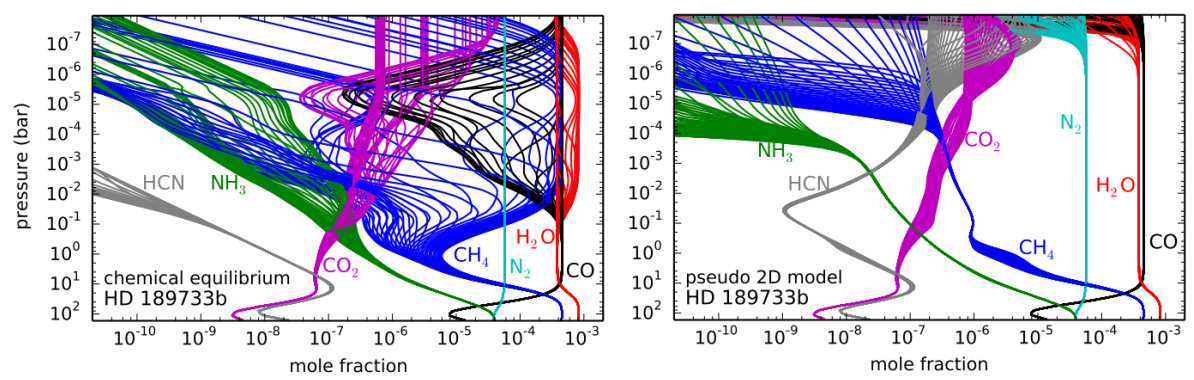

Fig. 21 Vertical abundances profiles for the atmosphere of the hot Jupiter HD 189733b. The composition corresponding to the thermochemical equilibrium is on the left. The composition calculated with the pseudo $2 \mathrm{D}$ model is on the right. For each species, the different lines correspond to different longitudes spanning the $0-360^{\circ}$ range. Adapted from Agúndez et al (2014a), reproduced with permission (C)ESO.

Planetary spectra are usually computed with $1 \mathrm{D}$ radiative-transfer codes. Because of the nature of these models, the longitudinal/latitudinal variations of the 
temperature and the chemical composition are neglected. Agúndez et al (2014a) investigate in which extent these longitudinal variations can affect the emission and transmission spectra. Because the chemical composition is horizontally quite homogenized, they find that the variability of composition with phase does not affect the emission spectrum, neither the transmission spectrum. The differences between the ingress and egress transmission spectra on one side, and the emission spectra at different phases on the other side are in reality due to the temperature variations (see Figs. 18 and 19 in Agúndez et al 2014a). The only change due to the chemical composition gradient is observed around $4.3 \mu \mathrm{m}$ and is due to the variation of $\mathrm{CO}_{2}$ abundance.

To estimate if the changes of the planetary spectra with phase can be detected with ARIEL we calculated the emission spectra corresponding to the morning, day, evening, and night sides, as well as the transmission spectra corresponding to the morning and evening limbs (Fig. 22). We binned them to the resolution of ARIEL
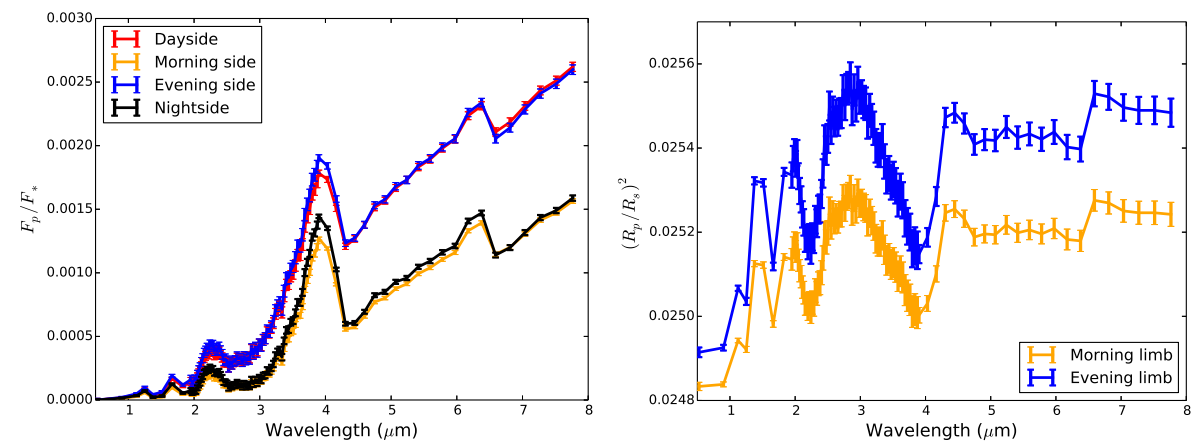

Fig. 22 Synthetic emission (left) and transmission (right) spectra for HD 189733b, with the chemical compositions calculated by the pseudo 2D model. They are binned to ARIEL resolutions, as explained in Table 1 . The error bars correspond to a SNR of 14 .

following the characteristics of Table 1] For secondary eclipse, one can observe two groups of emission spectra: the first one is made of the morning and night sides, and the second one gathers the day and evening sides. These two groups can be easily differentiated with one single observation, which corresponds to a SNR of 7 in the case of HD 189733b (see Table 2). However, it is more difficult to disentangle the two components of each groups because they are very close. The feature around $4 \mu \mathrm{m}$ can be identified with a SNR of 14 (i.e. four observations), but elsewhere it remains difficult to separate the spectra of each group.

For primary eclipse observations, we found that the transmission spectra of the morning and evening limbs are differentiable on the overall spectral range from a SNR of 7 (i.e. one single observation). Regarding these results, it is certain that ARIEL will allow us to better understand hot Jupiters, as it will have the technical capacity to determine horizontal and vertical variations of the thermal structure of their atmospheres. 


\section{Need for experimental data at high temperature}

Whatever the degree of sophistication of the atmospheric kinetics model (one dimension with constant thermal profile or chemistry-consistent thermal profile, two dimensions, and probably in the future three dimensions), of fundamental importance is to use data corresponding to the properties of this system. A model will always provide a result, and conclusions are then very easy to draw. However, if the input data used in the model are wrong, conclusions will be false, however advanced the model is.

With respect to the chemistry calculations, the term "input data" includes all the data necessary to calculate kinetics and photodissociations: reaction rates, absorption cross-sections, and quantum yields. Concerning the reaction rates, it has already been presented in Section 2 that, taking advantage of decades of intensive work in the field of combustion, Venot et al (2012) and Venot et al (2015) developed chemical schemes adapted to high temperature and validated experimentally.

But concerning absorption cross-sections and quantum yields, no such work exists already. Currently, all chemical models dealing with high temperatures don't have another choice but to use data at ambient temperature (or at 350-400 K in the best cases). Thus, an important uncertainty exists in the modelling of photochemistry.

In this context, an ambitious project has been developed at the Laboratoire Interuniversitaire des Systèmes Atmosphériques (France). It consists of measuring the absorption cross-sections of the most important species of planetary atmospheres at temperatures relevant for exoplanets. Thanks to an experimental setup that enables the gas to be heated to high temperatures (currently up to $1000 \mathrm{~K}$ ), measurements are being performed in synchrotron facilities (BESSY, Germany or SOLEIL, France). The absorption cross-section of carbon dioxide, $\sigma_{\mathrm{CO} 2}$, has been studied by Venot et al (2013) and Venot et al (2018) in the range [115-230] nm, up to $800 \mathrm{~K}$. The increase of the absorption together with the temperature is spectacular (see Fig. 23). Moreover, the use of this more accurate data in atmospheric models significantly affects the predicted chemical composition (see Fig. 24 . The abundance of $\mathrm{CO}_{2}$ is modified mainly in two regions: around $10^{-3}$ mbar and around 10 mbar. Between these two regions, the destruction of $\mathrm{CO}_{2}$ by photolysis is compensated by the production of $\mathrm{CO}_{2}$ through the reaction $\mathrm{CO}+\mathrm{OH}$ $\longrightarrow \mathrm{CO}_{2}+\mathrm{H}$. Changing only the absorption cross section of $\mathrm{CO}_{2}$, many species see their abundances varying by several orders of magnitude $\left(\mathrm{NH}_{3}, \mathrm{H}, \mathrm{CH}_{4}, \ldots\right)$. This confirms the urgency of acquiring a complete database of cross-sections at high temperature.

In Venot et al (2018), the authors also determine a parametrization of the absorption cross section of $\mathrm{CO}_{2}$ that permits to calculate the absorption of this molecule (more exactly the continuum of the absorption) at any temperature in the wavelength range [115-230] nm. Thus, they estimate the absorption of $\mathrm{CO}_{2}$ at $1500 \mathrm{~K}$ and use this data to study a very hot atmosphere (i.e. $\mathrm{T}=1500 \mathrm{~K}$ between $10^{-4}$ and $10 \mathrm{mbar}$ ). They find that in this very hot atmosphere, the contribution of photodissociations to the chemical composition is smaller than in cooler atmospheres. Consequently, even if the absorption of $\mathrm{CO}_{2}$ is much more important at $1500 \mathrm{~K}$ than at $800 \mathrm{~K}$, using $\sigma_{\mathrm{CO} 2}(1500 \mathrm{~K})$ instead of $\sigma_{\mathrm{CO} 2}(300 \mathrm{~K})$ generates less variations than in the atmosphere at $800 \mathrm{~K}$ (see Fig. 25). Variations of the chemical composition induced by the change of VUV absorption cross section 


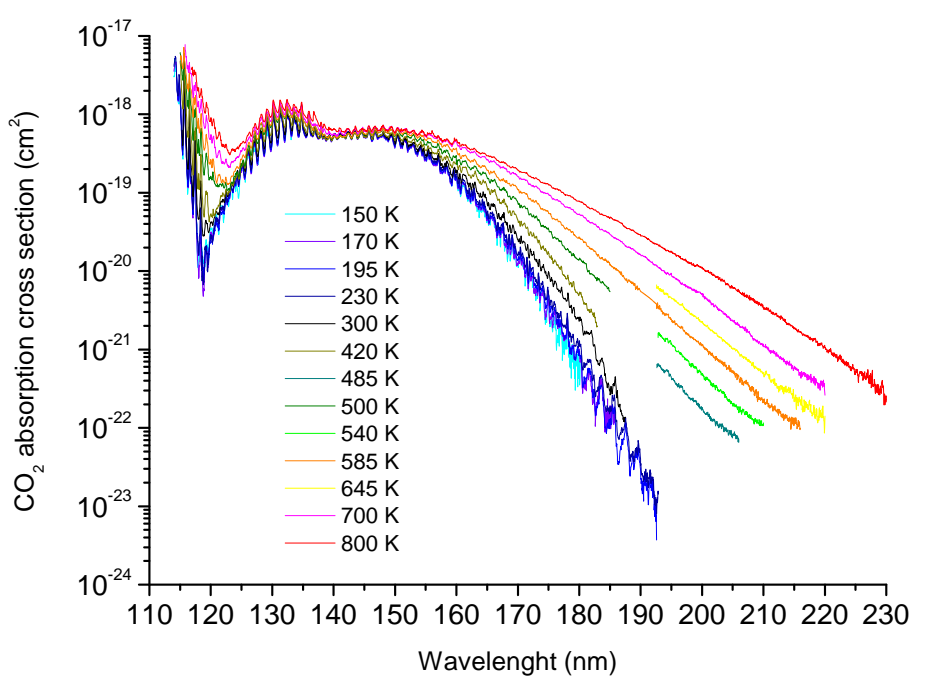

Fig. 23 VUV absorption cross sections of carbon dioxide at different temperatures from 150 to $800 \mathrm{~K}$. Adapted from Venot et al (2013, 2018), reproduced with permission (C)ESO.
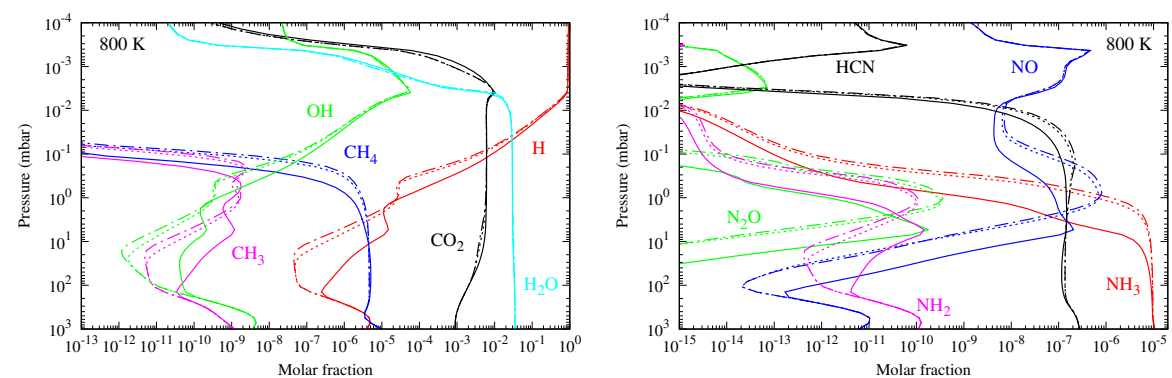

Fig. 24 Vertical abundances of several species when using the absorption cross section of $\mathrm{CO}_{2}$ at $300 \mathrm{~K}$ (full line) or at $800 \mathrm{~K}$ (dotted line). The atmospheric temperature between $10^{-4}$ and $10 \mathrm{mbar}$ is $800 \mathrm{~K}$. The dot-dashed lines correspond to a model using an analytical formula for the absorption of $\mathrm{CO}_{2}$ determined by Venot et al (2018). From Venot et al (2018), reproduced with permission (C)ESO.

in the photochemical model have noticeable consequences on the transmission spectrum in the case of a $800 \mathrm{~K}$ atmosphere only. High-resolution transmission spectra are shown in Venot et al (2018). Here we recalculated these spectra and binned them to the resolution of ARIEL assuming a signal-to-noise of 20. To estimate the noise, we took the characteristics of the star HD 128167 (F star, $\mathrm{T}_{\text {eff }}=6600 \mathrm{~K}, \mathrm{~d}=15.83 \mathrm{pc}, \operatorname{mag}_{k}=6$ ) since its stellar flux has been used in the photochemical model. Venot et al (2018) showed that the spectra corresponding to the models using the $\mathrm{CO}_{2}$ absorption cross section at $300 \mathrm{~K}$ or at $800 \mathrm{~K}$ present differences at three locations: at 2.7, 4.3, and $14.9 \mu \mathrm{m}$. These differences are quite 

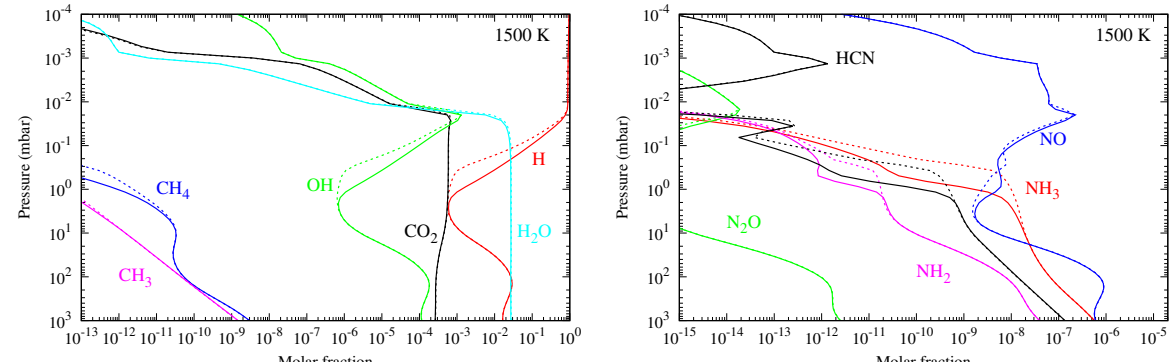

Fig. 25 Vertical abundances of several species when using the absorption cross section of $\mathrm{CO}_{2}$ at $300 \mathrm{~K}$ (full line) or at $1500 \mathrm{~K}$ (dotted line). The absorption at $1500 \mathrm{~K}$ is calculated with an analytical formula. The atmospheric temperature between $10^{-4}$ and 10 mbar is 800 K. From Venot et al (2018), reproduced with permission (c)ESO.

small, but the departure at 4.3 is distinguishable on ARIEL observations if they are performed with a SNR $\geq 20$ (see Fig 26). The range around $14.9 \mu \mathrm{m}$ will not be observed by ARIEL.

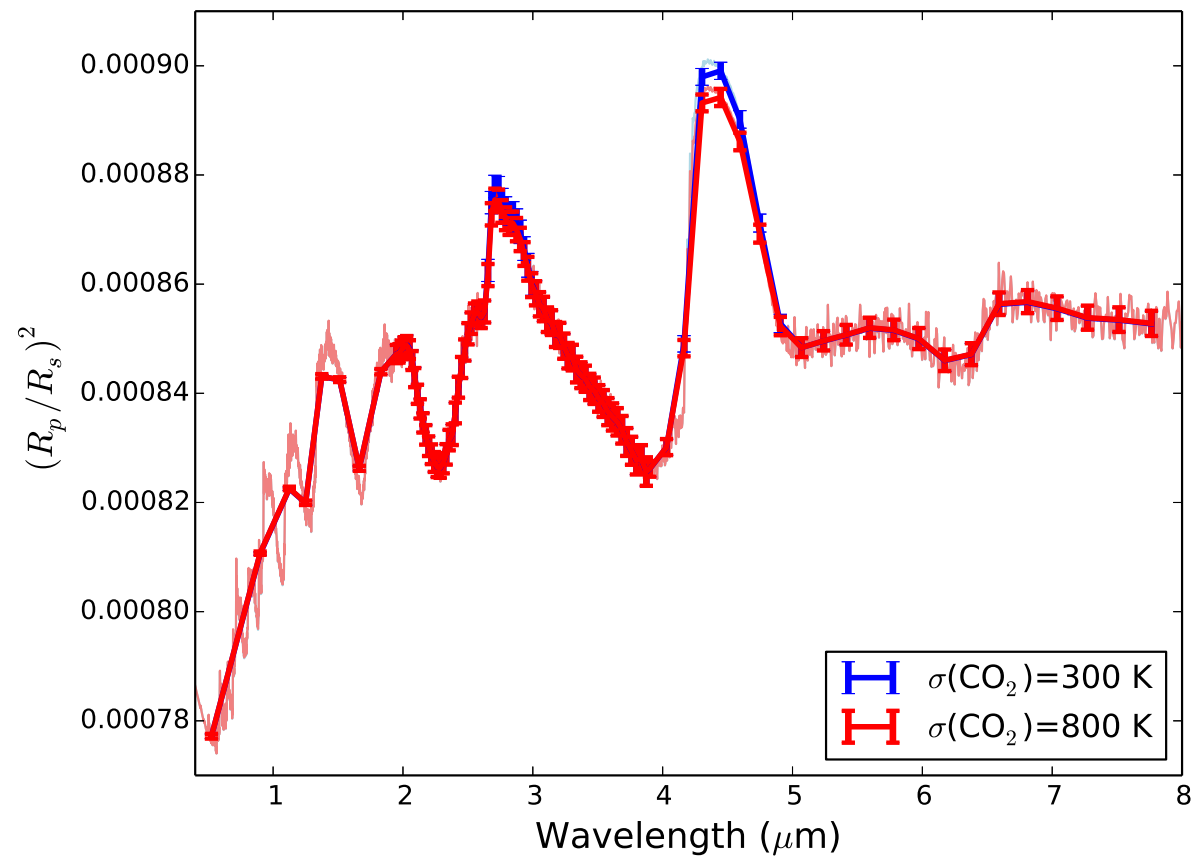

Fig. 26 Synthetic transmission spectra of the atmosphere at $800 \mathrm{~K}$ corresponding to the models using the absorption cross section of $\mathrm{CO}_{2}$ at $300 \mathrm{~K}$ (blue) or at $800 \mathrm{~K}$ (red). The bold spectra are binned to ARIEL resolution, as explained in Table 1 The error bars correspond to a SNR of 20. The fainter spectra have a higher resolution $(R=300)$, constant in wavelength. 
In view of these results, it appears more urgent to study other molecules at temperatures up to $\sim 1000 \mathrm{~K}$ rather than performing measurements at higher temperatures. Measurements of other species are in progress, such as $\mathrm{NH}_{3}, \mathrm{C}_{2} \mathrm{H}_{2}$, and HCN, and should be released soon.

\section{Chemical modeling and ARIEL}

Whilst significant developments are still required, the current state-of-the-art models provide us with an understanding of how various parameters and processes (temperature, metallicity, mixing, etc.) can affect the atmospheric chemical compositions of hot exoplanets. Indeed, for a given atmosphere it is possible to determine whether the atmosphere is likely to be described by chemical equilibrium or whether non-equilibrium processes will have a significant effect. If non-equilibrium effects are likely, then the use of kinetics models can predict the abundances of the chemical species and investigate the effect of these non-equilibrium abundances on the observable spectra. We have shown in this manuscript that the high precision of ARIEL will be able to discriminate relatively easily (with one or a few tens observations) the different assumptions that can be done of atmospheres (metallicity, $\mathrm{C} / \mathrm{O}$ ratio, temperature, etc.).

The limiting factor to a wider and deeper knowledge of exoplanet atmospheres is currently the limited precision, resolution and amount of observations. The coming of next-generation instruments will provide much higher precision spectra, which will constrain the models to a higher degree. However, the number of observations which can be made with a finite amount of telescope time is strictly limited. Therefore, ARIEL is eagerly awaited as it is the only project which can deliver a large survey of atmospheric spectra. Such a database of observed spectra for a large number of planets with different (and similar) properties is crucial to understanding how the planet properties, stellar type and orbital configurations alter the atmospheric chemistry; and of course, this understanding can be only be achieved by combining theory and observations.

By studying the chemical composition of the atmospheres of exoplanets, we can hope to gain some insight into the bulk compositions of such planets and eventually to their formation and evolution histories. The huge diversity of exoplanets discovered to date indicates that such a problem can only be tackled by considering a large sample of planets, which ARIEL will provide. We will finally be able to understand why so many exoplanetary systems currently detected appear radically different to our own Solar system, and indeed, to determine whether the Earth and the Solar system are truly unique.

Acknowledgements All figures extracted from previous publications have been reproduced with permission. The authors deeply thank the anonymous referee for his/her comments that greatly improve the manuscript. O. V. thanks the CNRS/INSU Programme National de Planétologie (PNP) for funding support. B.D. acknowledges funding from the European Research Council (ERC) under the European Unions Seventh Framework Programme (FP7/20072013) / ERC grant agreement no. 336792. I.P.W. acknowledges funding from the European Research Council (ERC) under the European Unions Seventh Framework Programme (FP7/20072013) / ERC grant agreement no. 758892. T. Z. is supported by the European Research Council (ERC) project ExoLights (617119) and from INAF trough the "Progetti Premiali" funding scheme of the Italian Ministry of Education, University, and Research. Y.M. greatly appreciates the CNES post-doctoral fellowship program and support for travel funding. 


\section{References}

Agúndez M, Venot O, Iro N, Selsis F, Hersant F, Hébrard E, Dobrijevic M (2012) The impact of atmospheric circulation on the chemistry of the hot Jupiter HD 209458b. Astronomy \& Astrophysics 548:A73, DOI 10.1051/0004-6361/ 201220365

Agúndez M, Parmentier V, Venot O, Hersant F, Selsis F (2014a) Pseudo 2D chemical model of hot-Jupiter atmospheres: application to HD 209458b and HD 189733b. Astronomy \& Astrophysics 564:A73, DOI 10.1051/0004-6361/ 201322895

Agúndez M, Venot O, Selsis F, Iro N (2014b) The Puzzling Chemical Composition of GJ 436b's Atmosphere: Influence of Tidal Heating on the Chemistry. The Astrophysical Journal 781:68, DOI 10.1088/0004-637X/781/2/68

Allard F, Hauschildt PH, Alexander DR, Tamanai A, Schweitzer A (2001) The Limiting Effects of Dust in Brown Dwarf Model Atmospheres. The Astrophysical Journal 556:357, DOI 10.1086/321547

Barman T (2007) Identification of Absorption Features in an Extrasolar Planet Atmosphere. The Astrophysical Journal Letters 661:L191-L194, DOI 10.1086/ 518736, 0704.1114

Burrows A, Sharp C (1999) Chemical equilibrium abundances in brown dwarf and extrasolar giant planet atmospheres. The Astrophysical Journal 512:843

Burrows A, Hubeny I, Budaj J, Knutson H, Charbonneau D (2007) Theoretical spectral models of the planet hd 209458b with a thermal inversion and water emission bands. The Astrophysical Journal Letters 668:L171

Burrows A, Budaj J, Hubeny I (2008) Theoretical spectra and light curves of close-in extrasolar giant planets and comparison with data. The Astrophysical Journal 678:1436

Cavalié T, Venot O, Selsis F, Hersant F, Hartogh P, Leconte J (2017) Thermochemistry and vertical mixing in the tropospheres of Uranus and Neptune: How convection inhibition can affect the derivation of deep oxygen abundances. Icarus 291:1-16, DOI 10.1016/j.icarus.2017.03.015

Cooper CS, Showman AP (2006) Dynamics and Disequilibrium Carbon Chemistry in Hot Jupiter Atmospheres, with Application to HD 209458b. The Astrophysical Journal 649:1048-1063, DOI 10.1086/506312

Drummond B, Tremblin P, Baraffe I, Amundsen DS, Mayne NJ, Venot O, Goyal J (2016) The Effects of Consistent Chemical Kinetics Calculations on the Pressure-Temperature Profiles and Emission Spectra of Hot Jupiters. ArXiv e-prints 1607.04062

France K, Froning CS, Linsky ea J L (2013) The ultraviolet radiation environment around $\mathrm{m}$ dwarf exoplanet host stars. The Astrophysical Journal 763:149

France K, Parke Loyd RO, Youngblood A, Brown A, Schneider PC, Hawley SL, Froning CS, Linsky JL, Roberge A, Buccino AP, Davenport JRA, Fontenla JM, Kaltenegger L, Kowalski AF, Mauas PJD, Miguel Y, Redfield S, Rugheimer S, Tian F, Vieytes MC, Walkowicz LM, Weisenburger KL (2016) The muscles treasury survey. i. motivation and overview. The Astrophysical Journal 820:89

Goldreich P, Soter S (1966) Q in the Solar System. Icarus 5:375-389, DOI 10. 1016/0019-1035(66)90051-0

Guillot T (2010) On the radiative equilibrium of irradiated planetary atmospheres. Astronomy \& Astrophysics 520:13, DOI 10.1051/0004-6361/200913396 
Heng K, Lyons JR (2016) Carbon Dioxide in Exoplanetary Atmospheres: Rarely Dominant Compared to Carbon Monoxide and Water in Hot, Hydrogendominated Atmospheres. The Astrophysical Journal 817:149, DOI 10.3847/ 0004-637X/817/2/149

$\mathrm{Hu}$ R, Seager S, Bains W (2012) Photochemistry in Terrestrial Exoplanet Atmospheres. I. Photochemistry Model and Benchmark Cases. The Astrophysical Journal 761:166, DOI 10.1088/0004-637X/761/2/166

Hut P (1981) Tidal evolution in close binary systems. Astronomy \& Astrophysics 99:126-140

Kopparapu Rk, Kasting JF, Zahnle KJ (2012) A photochemical model for the carbon-rich planet wasp-12b. The Astrophysical Journal 745:77

Kopparapu Rk, Ramirez RM, SchottelKotte Jea (2014) Habitable zones around main-sequence stars: Dependence on planetary mass. The Astrophysical Journal Letters 787:L29

Koskinen TT, Aylward AD, Smith CGA, Miller S (2007) A Thermospheric Circulation Model for Extrasolar Giant Planets. The Astrophysical Journal 661:515526, DOI 10.1086/513594

Kurucz RL (1979) Model atmospheres for g, f, a, b, and o stars. ApJS 40:1-340, DOI $10.1086 / 190589$

Lavvas P, Koskinen T, Yelle RV (2014) Electron Densities and Alkali Atoms in Exoplanet Atmospheres. The Astrophysical Journal 796:15, DOI 10.1088/ 0004-637X/796/1/15

Leconte J, Chabrier G, Baraffe I, Levrard B (2010) Is tidal heating sufficient to explain bloated exoplanets? Consistent calculations accounting for finite initial eccentricity. Astronomy \& Astrophysics 516:A64, DOI 10.1051/0004-6361/ 201014337

Lewis NK, Showman AP, Fortney JJ, Marley MS, Freedman RS, Lodders K (2010) Atmospheric Circulation of Eccentric Hot Neptune GJ436b. The Astrophysical Journal 720:344-356, DOI 10.1088/0004-637X/720/1/344

Liang MC, Parkinson CD, Lee AYT, Yung YL, Seager S (2003) Source of Atomic Hydrogen in the Atmosphere of HD 209458b. The Astrophysical Journal Letters 596:L247-L250, DOI 10.1086/379314

Liang MC, Seager S, Parkinson CD, Lee AYT, Yung YL (2004) On the Insignificance of Photochemical Hydrocarbon Aerosols in the Atmospheres of Close-in Extrasolar Giant Planets. The Astrophysical Journal Letters 605:L61-L64, DOI 10.1086/392509

Line MR, Liang MC, Yung YL (2010) High-temperature photochemistry in the atmosphere of hd 189733b. The Astrophysical Journal 717:496-502, DOI 10. 1088/0004-637X/717/1/496

Madhusudhan N (2012) C/O Ratio as a Dimension for Characterizing Exoplanetary Atmospheres. The Astrophysical Journal 758:36, DOI 10.1088/0004-637X/ $758 / 1 / 36$

Madhusudhan N, Mousis O, Johnson TV, Lunine JI (2011) Carbon-rich Giant Planets: Atmospheric Chemistry, Thermal Inversions, Spectra, and Formation Conditions. The Astrophysical Journal 743:191, DOI 10.1088/0004-637X/743/ $2 / 191$

Madhusudhan N, Agúndez M, Moses JI, Hu Y (2016) Exoplanetary Atmospheres - Chemistry, Formation Conditions, and Habitability. Space Science Reviews 205:285-348, DOI 10.1007/s11214-016-0254-3 
Miguel Y, Kaltenegger L (2014) Exploring atmospheres of hot mini-neptunes and extrasolar giant planets orbiting different stars with application to hd 97658b, wasp-12b, corot-2b, xo-1b, and hd 189733b. The Astrophysical Journal 780:166

Miguel Y, Kaltenegger L, Linsky JL, Rugheimer S (2015) The effect of lyman $\alpha$ radiation on mini-neptune atmospheres around $\mathrm{m}$ stars: application to gj 436b. Monthly Notices of the Royal Astronomical Society 446:345

Mollière P, van Boekel R, Dullemond C, Henning T, Mordasini C (2015) Model Atmospheres of Irradiated Exoplanets: The Influence of Stellar Parameters, Metallicity, and the C/O Ratio. The Astrophysical Journal 813:47, DOI 10.1088/0004-637X/813/1/47

Moses JI, Visscher C, Fortney JJ, Showman AP, Lewis NK, Griffith CA, Klippenstein SJ, Shabram M, Friedson AJ, Marley MS, Freedman RS (2011) Disequilibrium Carbon, Oxygen, and Nitrogen Chemistry in the Atmospheres of HD 189733b and HD 209458b. The Astrophysical Journal 737:15, DOI 10.1088/0004-637X/737/1/15

Moses JI, Madhusudhan N, Visscher C, Freedman RS (2013) Chemical Consequences of the $\mathrm{C} / \mathrm{O}$ Ratio on Hot Jupiters: Examples from WASP-12b, CoRoT-2b, XO-1b, and HD 189733b. The Astrophysical Journal 763:25, DOI 10.1088/0004-637X/763/1/25

Parmentier V, Guillot T (2014) A non-grey analytical model for irradiated atmospheres. i: Derivation. Astronomy \& Astrophysics 562

Parmentier V, Showman AP, Lian Y (2013) 3d mixing in hot jupiters atmospheres. i. application to the day/night cold trap in hd 209458b. Astronomy \& Astrophysics 558:A81

Prinn RG, Barshay SS (1977) Carbon monoxide on Jupiter and implications for atmospheric convection. Science 198:1031-1034, DOI 10.1126/science.198.4321. 1031

Puig L, Isaak K, Linder M, Escudero I, Crouzet PE, Walker R, Ehle M, Hübner J, Timm R, de Vogeleer B, Drossart P, Hartogh P, Lovis C, Micela G, Ollivier M, Ribas I, Snellen I, Swinyard B, Tinetti G, Eccleston P (2015) The phase 0/A study of the ESA M3 mission candidate EChO. Experimental Astronomy 40:393-425, DOI 10.1007/s10686-014-9419-9

Rimmer PB, Helling C (2016) A Chemical Kinetics Network for Lightning and Life in Planetary Atmospheres. The Astrophysical Journal Supplement Series 224:9, DOI 10.3847/0067-0049/224/1/9

Rocchetto M, Waldmann IP, Venot O, Lagage PO, Tinetti G (2016) Exploring Biases of Atmospheric Retrievals in Simulated JWST Transmission Spectra of Hot Jupiters. The Astrophysical Journal 833:120, DOI 10.3847/1538-4357/833/ $1 / 120$

Rothman L, Gordon I, Barber R, Dothe H, Gamache R, Goldman A, Perevalov V, Tashkun S, Tennyson J (2010) Hitemp, the high-temperature molecular spectroscopic database. Journal of Quantitative Spectroscopy and Radiative Transfer 111(15):2139-2150

Rothman LS, Gordon IE, Barbe A, Benner DC, Bernath PF, Birk M, Boudon V, Brown LR, Campargue A, Champion JP, Chance K, Coudert LH, Dana V, Devi VM, Fally S, Flaud JM, Gamache RR, Goldman A, Jacquemart D, Kleiner I, Lacome N, Lafferty WJ, Mandin JY, Massie ST, Mikhailenko SN, Miller CE, Moazzen-Ahmadi N, Naumenko OV, Nikitin AV, Orphal J, Perevalov VI, Perrin A, Predoi-Cross A, Rinsland CP, Rotger M, Šimečková M, Smith MAH, Sung K, 
Tashkun SA, Tennyson J, Toth RA, Vandaele AC, Vander Auwera J (2009) The HITRAN 2008 molecular spectroscopic database. JQSRT 110:533-572, DOI 10.1016/j.jqsrt.2009.02.013

Rothman LS, Gordon IE, Babikov Y, Barbe A, Chris Benner D, Bernath PF, Birk M, Bizzocchi L, Boudon V, Brown LR, Campargue A, Chance K, Cohen EA, Coudert LH, Devi VM, Drouin BJ, Fayt A, Flaud JM, Gamache RR, Harrison JJ, Hartmann JM, Hill C, Hodges JT, Jacquemart D, Jolly A, Lamouroux J, Le Roy RJ, Li G, Long DA, Lyulin OM, Mackie CJ, Massie ST, Mikhailenko S, Müller HSP, Naumenko OV, Nikitin AV, Orphal J, Perevalov V, Perrin A, Polovtseva ER, Richard C, Smith MAH, Starikova E, Sung K, Tashkun S, Tennyson J, Toon GC, Tyuterev VG, Wagner G (2013) The HITRAN2012 molecular spectroscopic database. JQSRT 130:4-50, DOI 10.1016/j.jqsrt.2013.07.002

Rugheimer S, Kaltenegger S, Zsom Aea (2013) Spectral fingerprints of earth-like planets around fgk stars. Astrobiology 13:251

Sarkar S, Papageorgiou A, Pascale E (2016) Exploring the potential of the ExoSim simulator for transit spectroscopy noise estimation. In: Space Telescopes and Instrumentation 2016: Optical, Infrared, and Millimeter Wave, Proceedings of the SPIE, vol 9904, p 99043R, DOI 10.1117/12.2234216

Seager S, Sasselov D (2000) Theoretical transmission spectra during extrasolar giant planet transits. The Astrophysical Journal 537:916

Sharp C, Burrows A (2007) Atomic and molecular opacities for brown dwarf and giant planet atmospheres. The Astrophysical Journal Supplement Series 168:140

Southworth J (2010) Homogeneous studies of transiting extrasolar planets - III. Additional planets and stellar models. Monthly Notices of the Royal Astronomical Society 408:1689-1713, DOI 10.1111/j.1365-2966.2010.17231.x

Tennyson J, Yurchenko SN (2012) ExoMol: molecular line lists for exoplanet and other atmospheres. MNRAS 425:21-33, DOI 10.1111/j.1365-2966.2012.21440.x, 1204.0124

Tsai SM, Lyons JR, Grosheintz L, Rimmer PB, Kitzmann D, Heng K (2017) VULCAN: An Open-source, Validated Chemical Kinetics Python Code for Exoplanetary Atmospheres. The Astrophysical Journal Supplement Series 228:20, DOI 10.3847/1538-4365/228/2/20

Tsiaras A, Rocchetto M, Waldmann IP, Venot O, Varley R, Morello G, Damiano M, Tinetti G, Barton EJ, Yurchenko SN, Tennyson J (2016) Detection of an Atmosphere Around the Super-Earth 55 Cancri e. The Astrophysical Journal 820:99, DOI 10.3847/0004-637X/820/2/99

Venot O, Hébrard E, Agúndez M, Dobrijevic M, Selsis F, Hersant F, Iro N, Bounaceur R (2012) A chemical model for the atmosphere of hot Jupiters. Astronomy \& Astrophysics 546:A43, DOI 10.1051/0004-6361/201219310

Venot O, Fray N, Bénilan Y, Gazeau MC, Hébrard E, Larcher G, Schwell M, Dobrijevic M, Selsis F (2013) High-temperature measurements of VUV-absorption cross sections of $\mathrm{CO}_{2}$ and their application to exoplanets. Astronomy \& Astrophysics 551:A131, DOI 10.1051/0004-6361/201220945

Venot O, Agúndez M, Selsis F, Tessenyi M, Iro N (2014) The atmospheric chemistry of the warm Neptune GJ 3470b: Influence of metallicity and temperature on the $\mathrm{CH}_{4} / \mathrm{CO}$ ratio. Astronomy \& Astrophysics 562:A51, DOI 10.1051/0004-6361/201322485

Venot O, Hébrard E, Agúndez M, Decin L, Bounaceur R (2015) New chemical scheme for studying carbon-rich exoplanet atmospheres. Astronomy \& Astro- 
physics 577:A33, DOI 10.1051/0004-6361/201425311

Venot O, Rocchetto M, Carl S, Roshni Hashim A, Decin L (2016) Influence of Stellar Flares on the Chemical Composition of Exoplanets and Spectra. The Astrophysical Journal 830:77, DOI 10.3847/0004-637X/830/2/77

Venot O, Bénilan Y, Fray N, Gazeau MC, Lefèvre F, Es-sebbar E, Hébrard E, Schwell M, Bahrini C, Montmessin F, Lefèvre M, Waldmann IP (2018) VUVabsorption cross section of carbon dioxide from 150 to $800 \mathrm{~K}$ and applications to warm exoplanetary atmospheres. Astronomy \& Astrophysics 609:A34, DOI 10.1051/0004-6361/201731295

Wakelam V, Herbst E, Loison JC, Smith IWM, Chandrasekaran V, Pavone B, Adams NG, Bacchus-Montabonel MC, Bergeat A, Béroff K, Bierbaum VM, Chabot M, Dalgarno A, van Dishoeck EF, Faure A, Geppert WD, Gerlich D, Galli D, Hébrard E, Hersant F, Hickson KM, Honvault P, Klippenstein SJ, Le Picard S, Nyman G, Pernot P, Schlemmer S, Selsis F, Sims IR, Talbi D, Tennyson J, Troe J, Wester R, Wiesenfeld L (2012) A KInetic Database for Astrochemistry (KIDA). The Astrophysical Journal Supplement 199:21, DOI 10.1088/0067-0049/199/1/21

Waldmann IP, Rocchetto M, Tinetti G, Barton EJ, Yurchenko SN, Tennyson J (2015a) Tau-rex. ii. retrieval of emission spectra. The Astrophysical Journal 813(1):13

Waldmann IP, Tinetti G, Rocchetto M, Barton EJ, Yurchenko SN, Tennyson J (2015b) Tau-rex i: A next generation retrieval code for exoplanetary atmospheres. The Astrophysical Journal 802(2):107

Youngblood A, France K, Loyd PRO, Linsky J, Redfield S, Schneider CP, Wood BE, Brown A, Froning C, Miguel Y, Rugheimer S, Walkowicz L (2016) The muscles treasury survey ii: Intrinsic lyman alpha and extreme ultraviolet spectra of $\mathrm{k}$ and $\mathrm{m}$ dwarfs with exoplanets. The Astrophysical Journal, accepted (arXiv:160401032)

Zahnle K, Marley MS, Fortney JJ (2009a) Thermometric Soots on Warm Jupiters? ArXiv e-prints 0911.0728

Zahnle K, Marley MS, Freedman RS, Lodders K, Fortney JJ (2009b) Atmospheric Sulfur Photochemistry on Hot Jupiters. The Astrophysical Journal Letters 701:L20-L24, DOI 10.1088/0004-637X/701/1/L20 